\title{
POLYHEDRAL PRODUCTS AND FEATURES OF THEIR HOMOTOPY THEORY
}

\author{
A. BAHRI, M. BENDERSKY, AND F. R. COHEN
}

\begin{abstract}
A polyhedral product is a natural subspace of a Cartesian product that is specified by a simplicial complex. The modern formalism arose as a generalization of the spaces known as moment-angle complexes which were developed within the nascent subject of toric topology. This field, which began as a topological approach to toric geometry and aspects of symplectic geometry, has expanded rapidly in recent years. The investigation of polyhedral products and their homotopy theoretic properties has developed to the point where they are studied in various fields of mathematics far removed from their origin. In this survey, we provide a brief historical overview of the development of this subject, summarize many of the main results and describe applications.
\end{abstract}

\section{Contents}

1. Introduction 2

2. The origin of polyhedral products in toric topology 6

3. The introduction of moment-angle complexes 9

4. Moment-angle complexes as intersections of quadrics 11

5. The cohomology of moment-angle complexes 13

6. The exponentiation property of polyhedral products 15

7. Fibrations 17

8. Unstable and stable decompositions of the polyhedral product 19

9. Equivariance of the stable splitting and an application to number theory 23

10. The case that $A_{i}=*$ for all $i$

11. The cohomology of polyhedral products and a spectral sequence 28

12. A geometric approach to the cohomology of polyhedral products 33

13. Polyhedral products and the Golodness of monomial ideal rings 36

14. Higher Whitehead products and loop spaces 38

Acknowledgements 40

References

2010 Mathematics Subject Classification. Primary: 55P42, 55Q15, 52C35, 52B11, 35S22, 13F55; Secondary: 14M25, 14F45, 55U10, 55R20, 55N10.

Key words and phrases. Polyhedral product, moment-angle complex, cohomology, arrangements, stable splitting, simplicial wedge, Davis-Januszkiewicz space, Golodness, monomial ideal ring.

Supported in part by grant 426160 from Simons Foundation. 


\section{INTRODUCTION}

A polyhedral product is a natural topological subspace of a Cartesian product, determined by a simplicial complex $K$ and a family of pointed pairs of spaces $\left(X_{i}, A_{i}\right)$, one for each vertex of $K$. As noted in [126], in the special case that $A_{i}$ is a basepoint for $X_{i}$, the polyhedral product mediates between the product $X_{1} \times X_{2} \times \cdots \times X_{m}$ when $K$ is the full $(m-1)$-simplex, and the wedge $X_{1} \vee X_{2} \vee \cdots \vee X_{m}$ when $K$ consist of $m$ discrete points.

We begin with a brief historical note. The development of the theory of polyhedral products was guided by their inextricable link to spaces known as moment-angle manifolds which arose within the subject of toric topology. This subject, which incorporates ideas from geometry, symplectic geometry, combinatorics and commutative algebra, has now swelled well beyond its original confines as a topological generalization of toric geometry.

Toric Topology has precipitated investigations into new areas of manifold and orbifold theory, centered around toric actions, [52, 35, 37, 61, 9, 68, 75, 46, 111, 93, 19, 20], and the unpublished notes of N. Strickland, [123]. An excellent overview by V. Buchstaber and N. Ray is to be found in 38 .

The study of polyhedral products in particular has matured to the point that it extends now into a wide variety of fields of mathematics distant from its origin, as detailed in the table below. Consequently, the development of their homotopy theory has become useful in this context.

In order to be more specific, it is now necessary to give a definition. Begin by setting $[m]=\{1,2, \ldots, m\}$ and define a category $\mathcal{C}([\boldsymbol{m}])$ whose objects are pairs $(\underline{X}, \underline{A})$ where

$$
(\underline{X}, \underline{A})=\left\{\left(X_{1}, A_{1}\right),\left(X_{2}, A_{2}\right), \ldots,\left(X_{m}, A_{m}\right)\right\}
$$

is a family of based CW-pairs. A morphism

$$
\underline{f}:(\underline{X}, \underline{A}) \longrightarrow(\underline{Y}, \underline{B})
$$

consists of $m$ continuous maps $f_{i}: X_{i} \longrightarrow Y_{i}$ satisfying $f_{i}\left(A_{i}\right) \subset B_{i}$. Next, let $K$ be a simplicial complex on the vertex set $[m]$. We consider $K$ to be a category where the objects are the simplices of $K$ and the morphisms $d_{\sigma, \tau}$ are the inclusions $\sigma \subset \tau$.

Definition 1.1. Let $K$ be a simplicial complex on the vertex set $[m]$ as above. A polyhedral product is a functor $Z(K ;-): \mathcal{C}([\boldsymbol{m}]) \longrightarrow$ Top satisfying

$$
Z(K ;(\underline{X}, \underline{A})) \subseteq \prod_{i=1}^{m} X_{i}
$$

that is given as the colimit of a diagram $D: K \rightarrow C W_{*}$, where at each $\sigma \in K$, we set

$$
D(\sigma)=\prod_{i=1}^{m} W_{i}, \quad \text { where } \quad W_{i}=\left\{\begin{array}{lll}
X_{i} & \text { if } & i \in \sigma \\
A_{i} & \text { if } & i \in[m]-\sigma .
\end{array}\right.
$$


Though here, the colimit is a union given by

$$
Z(K ;(\underline{X}, \underline{A}))=\bigcup_{\sigma \in K} D(\sigma)
$$

the full colimit structure is used heavily in the development of the elementary theory of polyhedral products. Notice that when $\sigma \subset \tau$ then $D(\sigma) \subseteq D(\tau)$. In the case that $K$ itself is a simplex,

$$
Z(K ;(\underline{X}, \underline{A}))=\prod_{i=1}^{m} X_{i}
$$

Remark. When all the pairs $\left(X_{i}, A_{i}\right)$ are the same pair $(X, A)$, the family $(\underline{X}, \underline{A})$ is written simply as $(X, A)$. Notice that in this case $A u t(K)$ acts naturally on $Z(K ;(X, A))$.

In a way entirely similar to that above, a related space $\widehat{Z}(K ;(\underline{X}, \underline{A}))$, called the smash polyhedral product, is defined by replacing the Cartesian product everywhere in Definition 1.1 by the smash product. That is,

$$
\widehat{D}(\sigma)=\bigwedge_{i=1}^{m} W_{i} \text { and } \widehat{Z}(K ;(\underline{X}, \underline{A}))=\bigcup_{\sigma \in K} \widehat{D}(\sigma)
$$

with

$$
\widehat{Z}(K ;(\underline{X}, \underline{A})) \subseteq \bigwedge_{i=1}^{m} X_{i}
$$

Polyhedral products generalize the spaces called moment-angle complexes which were developed by V. Buchstaber and T. Panov [34] and correspond to the case

$$
\left(X_{i}, A_{i}\right)=\left(D^{2}, S^{1}\right), \quad i=1,2, \ldots, m .
$$

As mentioned above, the construction of moment-angle complexes originated within the subject of toric topology. Specifically, M. Davis and T. Januszkiewicz [52], constructed spaces $z$ as quotients of a product of an $m$-dimensional torus and an $n$-dimensional polytope which is simple, meaning that it satisfies a certain homogeneity condition. (See Section 2.) They were able to realize a class of toric manifolds as quotients of $z$ by free torus actions. The space $z$ was then reformulated as a moment-angle manifold by V. Buchstaber and T. Panov, [34].

The moment-angle complex construction (3.2) was extended to pairs $(X, A)$ replacing $\left(D^{2}, S^{1}\right)$ by V. Buchstaber and T. Panov [34], N. Strickland (unpublished), V. Baskakov [22], G. Denham and A. Suciu [55] and T. Panov [109]. The construction in the full generality of Definition 1.1 appeared in [10]. 
The table below lists a selection of current applications of polyhedral products.
$(\underline{X}, \underline{A})$
$Z(K ;(\underline{X}, \underline{A}))$
$\left(D^{2}, S^{1}\right)$
toric geometry and topology, arachnid mechanisms
$\left(D^{1}, S^{0}\right)$ surfaces, number theory, representation theory, linear programming
$\left(S^{1}, *\right)$ right-angled Artin groups, robotics
$\left(\mathbb{R P}^{\infty}, *\right)$ right-angled Coxeter groups
$\left(\mathbb{C}, \mathbb{C}^{*}\right)$ complements of coordinate arrangements
$\left(\underline{\mathbb{R}}^{n},\left(\underline{\mathbb{R}}^{n}\right)^{*}\right)$ complements of certain non-coordinate arrangements
$\left(\underline{\mathbb{C P}}^{\infty}, \underline{\mathbb{C P}}^{k}\right)$ monomial ideal rings
$(\underline{E G}, \underline{G})$ free groups and monodromy
$(\underline{B G}, *)$ combinatorics, aspherical spaces
$(\underline{P X}, \underline{\Omega X})$ homotopy theory, Whitehead products $\left(S^{2 k+1}, *\right)$ graph products, quadratic algebras

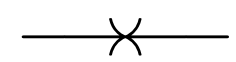

By way of motivation, we illustrate the Hopf map and the Whitehead product from the point of view of polyhedral products. The 3 -sphere $S^{3}$ is realized as a polyhedral product by taking $K$ to be the simplicial complex consisting of two discrete points,

$$
\partial\left(D^{2} \times D^{2}\right) \cong S^{1} \times D^{2} \cup_{S^{1} \times S^{1}} D^{2} \times S^{1}=Z\left(K ;\left(D^{2}, S^{1}\right)\right) \subset D^{2} \times D^{2} \subset \mathbb{C} \times \mathbb{C} .
$$

The action of the compact two-torus $T^{2}$ on the product $D^{2} \times D^{2}$ leaves invariant the component pieces: $S^{1} \times D^{2}, S^{1} \times S^{1}$ and $D^{2} \times S^{1}$, which comprise the basic building blocks of the polyhedral product, moment-angle complex, structure in this case. The Hopf map

$$
S^{3} \simeq S^{1} \times D^{2} \cup_{S^{1} \times S^{1}} D^{2} \times S^{1} \longrightarrow D^{2} \cup_{S^{1}} D^{2} \simeq S^{2}
$$

is the quotient by the diagonal subgroup $S^{1}$ in $T^{2}$ acting in a natural way.

Consider next the Whitehead product $[f, g]$ defined by

$$
S^{3} \stackrel{\omega}{\longrightarrow} S_{\alpha}^{2} \vee S_{\beta}^{2} \stackrel{f \vee g}{\longrightarrow} X
$$

where $\omega$ is a map attaching the top cell of $S^{2} \times S^{2}$ and $f: S_{\alpha}^{2} \longrightarrow X$ and $g: S_{\beta}^{2} \longrightarrow X$ are two basepoint preserving maps, [74, page 381]. The particular case $X=S_{\alpha}^{2} \vee S_{\beta}^{2}$ and $f=\iota_{\alpha}, g=\iota_{\beta}$, being the respective inclusions of $S_{\alpha}^{2}$ and $S_{\beta}^{2}$ into $S_{\alpha}^{2} \vee S_{\beta}^{2}$, gives the Whitehead product $\left[\iota_{\alpha}, \iota_{\beta}\right]$. (Note here that $\iota_{\alpha} \vee \iota_{\beta}$ is the identity map.) From the point of view of polyhedral products $1{ }^{1},\left[\iota_{\alpha}, \iota_{\beta}\right]$ is induced by the map of pairs $\left(D^{2}, S^{1}\right) \longrightarrow\left(S^{2}, *\right)$ which collapses $S^{1} \subset D^{2}$ to a point,

$$
S^{3} \simeq D_{\alpha}^{2} \times S_{\beta}^{1} \cup_{S_{\alpha}^{1} \times S_{\beta}^{1}} S_{\alpha}^{1} \times D_{\beta}^{2} \longrightarrow\left(S_{\alpha}^{2} \times *\right) \cup_{* \times *}\left(* \times S_{\beta}^{2}\right) \simeq S_{\alpha}^{2} \vee S_{\beta}^{2} .
$$

\footnotetext{
${ }^{1}$ Lukas Katthän alerted us to this fact.
} 
Here, the space on the right-hand side is the polyhedral product $Z\left(K ;\left(\underline{S}^{2}, *\right)\right)$ where again, $K$ consists of two discrete points. Within this subject, wedge products appear often, thus Whitehead products appear in profusion. These topics are discussed in more detail in Section 14 .

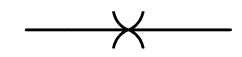

The paper is organized as follows. A brief technical discussion of the origin of polyhedral products as moment-angle complexes in Sections 2 and 3 , includes the original topological construction of toric manifolds by M. Davis and T. Januszkiewicz [52] and the reformulation of one of their main spaces as a moment-angle complex by V. Buchstaber and T. Panov, 34, 35.

A discussion follows in Section 4 about the independent discovery of moment-angle complexes as intersections of certain quadrics. We describe briefly the work of S. López de Medrano [100], S. López de Medrano and A. Verjovsky [101], F. Bosio and L. Meersseman [30], followed by that of S. López de Medrano and S. Gitler [65]. This approach has proved effective in identifying classes of moment-angle manifolds that are connected sums of products of spheres. This is followed in Section 5 by a sketch of the computation of the cohomology ring of moment-angle complexes.

We begin our focus on more general polyhedral products in Section 6, by describing their behaviour with respect to the exponentiation of CW-pairs. This has led to an application to the study of toric manifolds [58, 128, 129, 124, 70, 172, 45, and orbifolds [18]. An application to topological joins is included as an example of the utility of this property with respect to exponentiation.

The behaviour of polyhedral products with respect to fibrations, due to G. Denham and A. Suciu [55], is surveyed briefly in Section 7. We take advantage of the formalism to describe briefly the early work in this area by G. Porter [117, 118], T. Ganea [64] and A. Kurosh [92]. and G. W. Whitehead. Instances of polyhedral products appear also in the work of D. Anick [5].

In Section 8, we review the various fundamental unstable and stable splitting theorems for the polyhedral product. These theorems give access to the homotopy type of the polyhedral product in many of the most important cases and drive a number of applications. The identification of the various wedge summands which appear in the stable decompositions, occupies the second part of this section.

A fine theorem of A. Al-Raisi [2] shows that, in certain cases, the stable splitting of the polyhedral product can be chosen to be equivariant with respect to the action of Aut $(K)$. This and other related results, described in Section 9, represent the first foray of the subject into representation theory. An application of Al-Raisi's theorem to surfaces yields a cyclotomic identity. 
The important case $Z(K ;(\underline{X}, \underline{*}))$ is discussed in Section 10 . It arises in algebraic combinatorics, the study of free groups and monodromy representations, geometric group theory, right-angled Coxeter and Artin groups and asphericity,

In this section also, we use a recent result of T. Panov and S. Theriault [113] to give a short proof that if $K$ is a flag simplicial complex then, for a discrete group $G$, $Z(K ;(B G, *))$ is an Eilenberg-Mac Lane space, a known result, [43, 53, 122,

Results on the cohomology of polyhedral products are presented in Section 11. The discussion begins with a description of the cup-product structure as a consequence of the stable splitting Theorem 8.6. A spectral sequence corresponding to a natural filtration of $Z(K ;(\underline{X}, \underline{A}))$ by the simplices of $K$ is described next and followed by several examples.

Section 12 describes an entirely geometric approach to the calculation of the cohomology. The notion of wedge decomposable pairs is introduced and the corresponding polyhedral products are determined explicitly as wedges of identifiable spaces. Moreover, it is shown in Theorem 12.6, that over a field, every CW-pair $(\underline{X}, \underline{A})$ of finite type is cohomologically wedge decomposable. This suffices to give the additive structure of the homology of any $Z(K ;(X, A))$ for CW-pairs $(X, A)$ of finite type, and any field coefficients.

The application of polyhedral products to questions concerning the Golod properties of certain rings is described in Section 13 . Higher Whitehead products, constructed using polyhedral products, are discussed in Section 14. In cases when a moment-angle complex splits into a wedge of spheres, higher Whitehead products describe certain canonical maps related to the inclusions of the summands, [112, 71, 83, 1]. They arise also in the analysis of $\Omega Z\left(K ;\left(D^{2}, S^{1}\right)\right)$.

Though the $\mathrm{KO}$-theory of certain polyhedral products and toric manifolds is discussed in the literature [9, 6], it is omitted from this article. Applications to robotics $($ [76, 88], for example) are also omitted.

\section{ThE ORIGIN OF POLYHEDRAL PRODUCTS IN TORIC TOPOLOGY}

A polytope is the convex hull of a finite set of points in some $\mathbb{R}^{n}$. The dimension of the polytope is the dimension of its affine hull, [35, Chapter 1]. We shall assume that an $n$-dimensional polytope is a subset of $\mathbb{R}^{n}$. A codimension-one face of a polytope is called a facet. A polytope $P^{n}$ is called simple if at each vertex, exactly $n$ facets intersect. The convention is to let $m$ denote the number of facets of $P^{n}$.

In order to introduce the notion of a toric manifold, we recall that the real torus $T^{n}$ acts on $\mathbb{C}^{n}$ in a standard way, and the quotient is

$$
\mathbb{C}^{n} / T^{n} \cong \mathbb{R}_{+}^{n}=\left\{\left(x_{1}, \ldots, x_{n}\right) \in \mathbb{R}^{n}: x_{i} \geq 0 \text { for } i=1, \ldots, n\right\}
$$

A toric manifold $M^{2 n}$, (sometimes called a quasitoric manifold in the literature), is a compact manifold covered by local charts $\mathbb{C}^{n}$, with a global action of a real $n$-dimensional 
torus $T^{n}$, that restricts to the standard action on each $\mathbb{C}^{n}$ component. Under this action, each copy of $\mathbb{C}^{n}$ must have a quotient $\mathbb{C}^{n} / T^{n} \cong \mathbb{R}_{+}^{n}$ which is a neighbourhood of a vertex of a simple polytope $P^{n} \cong M^{2 n} / T^{n}$.

Remark. Smooth projective toric varieties are examples of toric manifolds in the sense above.

A topological approach to the construction of toric manifolds was developed by M. Davis and T. Januszkiewicz in [52]. They begin with a simple polytope $P^{n}$ and a function

$$
\lambda: \mathcal{F}=\left\{F_{1}, F_{2}, \ldots, F_{m}\right\} \longrightarrow \mathbb{Z}^{n} .
$$

from the set of facets of $P^{n}$ into an $n$-dimensional integer lattice. The function $\lambda$ must satisfy a regularity condition, namely, whenever $F=F_{i_{1}} \cap F_{i_{2}} \cap \cdots \cap F_{i_{l}}$ is a codimension$l$ face of $P^{n}$, then $\left\{\lambda\left(F_{i_{1}}\right), \lambda\left(F_{i_{2}}\right), \ldots, \lambda\left(F_{i_{l}}\right)\right\}$ spans an $l$-dimensional submodule of $\mathbb{Z}^{n}$ which is a direct summand. (The fact that every such face can be written uniquely as such an intersection is a consequence of the polytope being simple.) Such a map is called a characteristic function associated to $P^{n}$.

Next, regarding $\mathbb{R}^{n}$ as the Lie algebra of $T^{n}$, the map $\lambda$ is used to associate to each codimension- $l$ face $F$ of $P^{n}$ a rank- $l$ subgroup $G_{F}^{\lambda} \subset T^{n}$, namely the subgroup of $T^{n}$ determined by the span of the $\lambda\left(F_{i_{j}}\right)$. (Specifically, writing $\lambda\left(F_{i_{j}}\right)=\left(\lambda_{1 i_{j}}, \lambda_{2 i_{j}}, \ldots, \lambda_{n i_{j}}\right)$ gives

$$
G_{F}^{\lambda}=\left\{\left(e^{2 \pi i\left(\lambda_{1 i_{1}} t_{1}+\lambda_{1 i_{2}} t_{2}+\cdots+\lambda_{1 i_{l}} t_{l}\right)}, \ldots, e^{2 \pi i\left(\lambda_{n i_{1}} t_{1}+\lambda_{n i_{2}} t_{2}+\cdots+\lambda_{n i_{l}} t_{l}\right)}\right) \in T^{n}\right\}
$$

where $t_{i} \in \mathbb{R}, i=1,2, \ldots, l$.)

Finally, let $p \in P^{n}$ and $F(p)$ be the unique face with $p$ in its relative interior. Define an equivalence relation $\sim_{\lambda}$ on $T^{n} \times P^{n}$ by $(g, p) \sim_{\lambda}(h, q)$ if and only if $p=q$ and $g^{-1} h \in G_{F(p)}^{\lambda} \cong T^{l}$. Then

$$
M^{2 n} \cong M^{2 n}(\lambda)=T^{n} \times P^{n} / \sim_{\lambda}
$$

is a smooth, closed, connected, $2 n$-dimensional toric manifold with $T^{n}$ action induced by left translation [52, page 423]. A projection

$$
\pi: M^{2 n} \rightarrow P^{n} \cong M^{2 n} / T^{n}
$$

onto the polytope is induced from the projection $T^{n} \times P^{n} \rightarrow P^{n}$.

Example 2.1. Consider the case for which $P^{n}=[0,1]$, the one-simplex having dimension $n=1$ and two facets, so $m=2$. Here, the set of facets $\mathcal{F}=\left\{F_{1}, F_{2}\right\}$ consists of the two vertices of the one-simplex. Define $\lambda: \mathcal{F} \longrightarrow \mathbb{Z}^{1}$ by $\lambda\left(F_{1}\right)=-1$ and $\lambda\left(F_{2}\right)=1$. Topologically, $T^{1} \times P^{1}$ is the cylinder $T^{1} \times[0,1]$. In this case, the equivalence relation $\sim_{\lambda}$ collapses to points each circle $T^{1} \times\{0\}$ and $T^{1} \times\{1\}$, yielding

$$
T^{1} \times P^{1} / \sim_{\lambda} \cong S^{2}
$$


In fact, this $S^{2}$ has the structure of the toric variety $\mathbb{C P}^{1}$.

Remark. Every projective non-singular toric variety has the description $(2.2) ; P^{n}$ and $\lambda$ encode topologically the information in the defining fan, [36, Chapter 5].

As part of their approach to the construction of toric manifolds, the authors of [52] introduced a second manifold

$$
z=T^{m} \times P^{n} / \sim
$$

where $T^{m}$ denotes the real $m$-torus, its coordinate circles indexed by the $m$ facets of the simple polytope $P^{n}$. (Notice here a distinction between (2.3) and 2.2) given by the fact that $T^{m}$ has replaced $T^{n}$; the equivalence relation will be different too. This space was introduced into the theory of toric manifolds in [52] in order to facilitate the calculation of the cohomology of the toric manifold $M^{2 n}(\lambda)$ in (2.2).) The equivalence relation $\sim$ is given by defining $\theta: \mathcal{F} \longrightarrow \mathbb{Z}^{m}$ by $\theta\left(F_{i}\right)=\underline{e}_{i} \in \mathbb{Z}^{m}$, the standard basis vector.

Remark. Unlike a characteristic map $\lambda$ (above) the map $\theta$ indexes the coordinate circles in $T^{m}$ by the facets of $P^{n}$, and so depends on the combinatorics of the polytope $P^{n}$ only.

Next, by exact analogy with the construction of the subgroups $G_{F}^{\lambda} \subset T^{n}$ above for each codimension- $l$ face $F$ of $P^{n}$, the map $\theta$ determines a rank- $l$ subgroup $G_{F}^{\theta} \subset T^{m}$. The explicit description of this group is similar to (2.1). Finally, the equivalence relation $\sim$ is defined in a manner entirely analogous to $\sim_{\lambda}$ above. That is, for $p \in P^{n}$, and $F(p)$, the unique face with $p$ in its relative interior, define $\sim$ on $T^{n} \times P^{n}$ by $(g, p) \sim(h, q)$ if and only if $p=q$ and $g^{-1} h \in G_{F(p)}^{\theta} \cong T^{l}$.

It follows that $z$ is a smooth, closed, connected, $(m+n)$-dimensional manifold endowed with a $T^{m}$ action induced by left translation [52, page 423]. A projection $z \rightarrow z / T^{m} \cong P^{n}$ onto the polytope is induced from the projection $T^{m} \times P^{n} \rightarrow P^{n}$. (See also [7] and [87].)

Example 2.2. Consider the case for which $P^{n}$ is the one-simplex again so that $n=1$ and $m=2$. Topologically, $T^{m} \times P^{n}$ is the cylinder $T^{2} \times[0,1]$. The equivalence relation collapses one of the coordinate circles in $T^{2} \times\{0\}$ to a point as a group, as well as the other coordinate circle in $T^{2} \times\{1\}$. This gives

$$
z \cong S^{1} * S^{1} \cong S^{3} \text {. }
$$

A reformulation of the ideas above indicates that every toric manifold $M^{2 n}$ can be described as a quotient

$$
M^{2 n} \cong M^{2 n}(\lambda) \cong z / T^{m-n}
$$

where here, $T^{m-n} \subset T^{m}$ is the torus determined by the kernel of the map $\lambda$, which the regularity condition of $\lambda$ ensures acts freely. In the next section, the space $z$ is reformulated as a moment-angle manifold. 


\section{The introduction of MOMEnT-ANGLE COMPlEXES}

Every simple polytope $P^{n}$ with $m$ facets, embeds naturally into the union of the facets of the $m$-cube $I^{m}$ by virtue of its cubical decomposition, [35, Construction 4.5]. V. Buchstaber and T. Panov constructed a $T^{m}$-invariant natural subspace $Z_{K_{P^{n}}}$ of $\mathbb{C}^{m}$, that completes a commutative diagram:

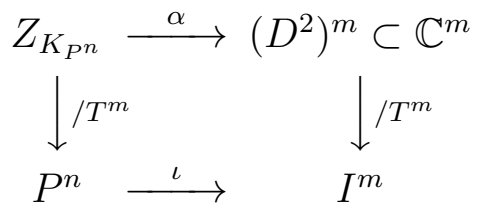

Here, $D^{2} \subset \mathbb{C}$ is the unit disc with the standard circle action, the vertical maps are quotients by the standard action of $T^{m}$, and $K_{P^{n}}$ denotes the boundary complex of the dual of $P$, (the vertices of $K_{P^{n}}$ are the facets of $P^{n}$ and simplices correspond to intersections of facets), [36, Example 2.2.4]. They recognized the space $Z_{K_{P^{n}}}$ as the natural subspace of $\left(D^{2}\right)^{m}$ defined as a colimit by a diagram $D: K_{P^{n}} \rightarrow C W_{*}$, which at each $\sigma \in K_{P^{n}}$, is given by

$$
D(\sigma)=\prod_{i=1}^{m} W_{i}, \quad \text { where } \quad W_{i}=\left\{\begin{array}{lll}
D^{2} & \text { if } \quad i \in \sigma \\
S^{1} & \text { if } \quad i \in[m]-\sigma .
\end{array}\right.
$$

Here, the colimit is $Z_{K_{P^{n}}}=\bigcup_{\sigma \in K_{P^{n}}} D(\sigma)$, and is now written $Z\left(K_{P^{n}} ;\left(D^{2}, S^{1}\right)\right)$; it was called a moment-angle manifold by V. Buchstaber and T. Panov.

In order to verify that the moment-angle complex $Z\left(K_{P^{n}} ;\left(D^{2}, S^{1}\right)\right)$ is in fact the space $z$ constructed by M. Davis and T. Januszkiewicz, we work locally. Construction (2.3) can be analyzed in the neighborhood of a vertex, where the simple polytope $P^{n}$ is PLhomeomorphic to $\mathbb{R}_{+}^{n}$. 


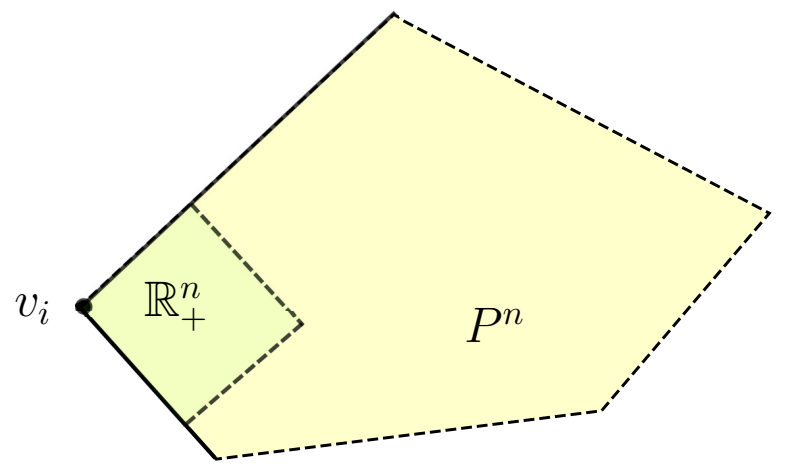

Figure 1. The local structure of a simple polytope $P^{n}$

The polytope can be given a cubical structure as in [35, Construction 5.8 and Lemma 6.6]. A cube $I^{n}$, anchored by the vertex $v_{i}$, sits inside the copy of $\mathbb{R}_{+}^{n}$ which arises by deleting all faces of $P^{n}$ which do not contain $v_{i}$. Locally, $T^{m} \times P^{n}$ is

$$
T^{m} \times I^{n} \cong\left(S^{1} \times I\right)^{n} \times\left(S^{1}\right)^{m-n} .
$$

Recall that the map $\theta$, defined in Section 2, indexes all the circles in $T^{m}$ by the facets of the polytope, so the order of factors here has been shuffled naturally. The factors $S^{1}$ which are paired with a copy of $I$ are those corresponding to the facets of $P^{n}$ which meet at $v_{i}$. The effect of the equivalence relation $\sim$ in $(2.3)$ on $T^{m} \times I^{n}$ is to convert every $S^{1} \times I$ on the right-hand side of $(3.3)$ into a disc $D^{2}$, by collapsing $S^{1} \times\{0\}$ to a point. So

$$
T^{m} \times I^{n} / \sim \cong\left(D^{2}\right)^{n} \times\left(S^{1}\right)^{m-n}
$$

The vertices of $P^{n}$ correspond to the maximal simplices of the simplicial complex $K_{P^{n}}$ so, assembling the blocks (3.4), gives the moment-angle manifold

$$
z=T^{m} \times P^{n} / \sim \cong Z\left(K_{P^{n}} ;\left(D^{2}, S^{1}\right)\right) .
$$

Remark 3.1. In a natural way, the Buchstaber-Panov formulation generalizes from $K_{P^{n}}$ to any simplicial complex $K$, but the result is not in general a manifold. For general $K$ the space $Z\left(K ;\left(D^{2}, S^{1}\right)\right)$ is called a moment-angle complex. The condition that $K$ be a polytopal sphere, (a triangulated sphere which is isomorphic to the boundary complex of a polytope which has all its faces simplices, a simplicial polytope, [36, Definition 2.5.7]), ensures that $Z\left(K ;\left(D^{2}, S^{1}\right)\right)$ is a manifold. This was weakened in [40, Corollary 2.10] to: the moment-angle complex is a topological $(n+m)$-manifold if and only if the realization of $K$ is a generalized homology $(n-1)$-sphere. The latter is defined to be a triangulated polyhedron, which has the homology of an $(n-1)$-sphere for which the link of each $p$-simplex has the homology of an $(n-2-p)$-sphere [40]. 
Currently, the best result implying that the moment-angle complex is a smooth manifold, requires that $K$ be the simplicial complex underlying a complete simplicial fan; more details are to be found in [115, Theorem 2.2] and [110, Theorem 9.2].

The construction of moment-angle complexes was extended to simplicial posets by Z. Lü and T. Panov in [102] where they are used to study the face rings of posets topologically.

Below, the 2-torus is exhibited as the polyhedral product $Z\left(K ;\left(D^{1}, S^{0}\right)\right) \subset\left(D^{1}\right)^{4}$, where $K$ is the boundary complex of a square on vertices $\{1,2,3,4\}$. According to Definition 1.1, the empty simplex of $K$ contributes $\left(S^{0}\right)^{4}$ which is identified with the 16 vertices of $\left(D^{1}\right)^{4}$, and the four vertices of $K$ contribute the one-skeleton consisting of 32 edges. The maximal simplices of $K$ are: $\sigma_{1}=\{12\}, \sigma_{2}=\{14\}, \sigma_{3}=\{23\}, \sigma_{4}=\{34\}$.

Remark. For clarity, we have included the whole one-skeleton in each $D\left(\sigma_{i}\right)$ below, but, strictly speaking, the whole one-skeleton should appear in the final picture only.

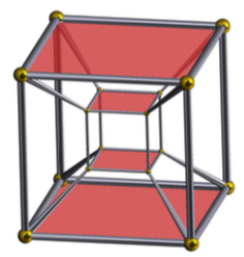

$D\left(\sigma_{1}\right)$

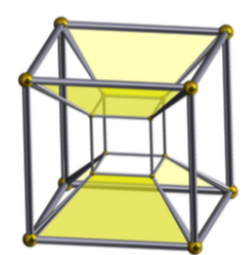

$D\left(\sigma_{2}\right)$

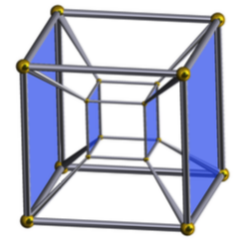

$D\left(\sigma_{3}\right)$

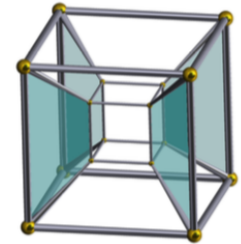

$D\left(\sigma_{4}\right)$

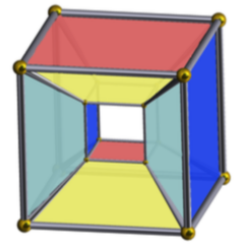

the torus

Figure 2. The 2-torus as the polyhedral product $Z\left(K ;\left(D^{1}, S^{0}\right)\right)$

(This figure is reproduced by kind permission of Alvise Trevisan, cf. [127].)

\section{Moment-Angle Complexes as Intersections of QUADrics}

The purpose of this section is to provide an overview of the independent topological development of moment-angle complexes arising from beautiful work of F. Bosio and L. Meersseman [30], Y. Barreto, S. López de Medrano and A. Verjovsky [21], S. López de Medrano [100], as well as his subsequent joint work with S. Gitler [65], and S. López de Medrano and A. Verjovsky [101], Closely related work was developed earlier, and independently, by C. T. C. Wall [130]. All of these authors considered quadrics specified by certain natural choices of homogeneous quadratic polynomials intersected with the unit sphere. One consequence is the López de Medrano-Gitler proof of a conjecture of BosioMeersseman in the case that the polytope $P^{n}$ is even dimensional and "dual neighborly". For such polytopes $P^{n}$, they show that $Z\left(K_{P^{n}} ;\left(D^{1}, S^{0}\right)\right)$ is a connected sum of products of spheres. In addition to the proof of this conjecture, the authors of [65] also give further computations of various cup-products in moment-angle manifolds.

Let $m$ and $n$ be positive integers satisfying $m>n$. Set $k=2 m-n-1$. A certain manifold, $M \subset \mathbb{C}^{m}$ associated to an $n$-dimensional polytope, is defined next as the intersection of $k$ quadrics. Specifically with $\lambda_{i} \in \mathbb{R}^{k}, i=1, \ldots, m$ the manifold $M$ is the 
intersection of the solution of a homogeneous system of equations with the unit sphere in $\mathbb{C}^{m}$,

$$
\left\{\left(z_{1}, z_{2}, \ldots, z_{m}\right) \in \mathbb{C}^{m}: \sum_{i=1}^{m} \lambda_{i}\left|z_{i}\right|^{2}=0\right\} \cap\left\{\left(z_{1}, z_{2}, \ldots, z_{m}\right) \in \mathbb{C}^{m}: \sum_{i=1}^{m}\left|z_{i}\right|^{2}=1\right\} .
$$

The manifold $\mathrm{M}$ admits an action of the $m$-torus $T^{m}$ by multiplication of unit complex numbers on each of the coordinates. The quotient by this action can be identified with the $n$-dimensional convex polytope $P^{n}$ given by

$$
\sum_{i=1}^{2 m} \lambda_{i} x_{i}=0, \quad \sum_{i=1}^{2 m} x_{i}=1, \quad x_{i} \geq 0 .
$$

This construction is essentially equivalent to the construction (2.3) of a moment-angle manifold from a simple polytope in Section 2. The simplicity of $P^{n}$ is implied by the weak hyperbolicity condition: the convex hull of any subset of $\left\{\lambda_{1}, \ldots, \lambda_{m}\right\}$, with $k$ or fewer elements does not contain the origin. The real points of this manifold correspond to the associated real moment-angle manifold $Z\left(K_{P^{n}} ;\left(D^{1}, S^{0}\right)\right)$ that has natural a $\mathbb{Z}_{2}^{n}$-action.

S. López de Medrano and A. Verjovsky [101] constructed a class of non-Kähler, compact, manifolds generalizing Hopf and Calabi-Eckmann manifolds that are projectivizations of some of these moment-angle manifolds. L. Meersseman [103], generalized this construction and also observed that all even-dimensional moment-angle manifolds are themselves in this class.

S. López de Medrano [100], showed that when $k=2$, the moment-angle manifolds constructed above and their real parts are either three-fold products of spheres or connected sums of two-fold products of spheres.

These works culminated in the article by S. Gitler and S. López de Medrano [65], showing that for all values of $k$, these manifolds are frequently connected sums of products of spheres.

One result is that if $K$ is a polytopal sphere, (see Remark 3.1) then any manifold $Z\left(K ;\left(D^{1}, S^{0}\right)\right)$ which is $2 c$-dimensional, and $(c-1)$-connected for $c \geq 3$, must be diffeomorphic to a connected sum of products of spheres. This is a proof of a conjecture of Bosio and Meersseman [30, page 115], in the case of even dimensional dual-neighborly polytopes mentioned earlier in this section. The authors also introduce operations including corner cutting or edge cutting of $K$ and they analyze the effect of these operations on the manifolds $Z\left(K ;\left(D^{1}, S^{0}\right)\right)$. One result in this direction is that they obtain new, infinite families of manifolds which are diffeomorphic to connected sums of products of spheres.

The methods in [65] influence the argument used by S. Theriault in [125] to prove Panov's Conjecture. The number of wedge summands in a homotopy decomposition of the moment-angle complex corresponding to the simplicial complex consisting of $l$ discrete 
points, is described in 8.1). Panov's conjecture relates this to the connected sum factors in a decomposition of the moment-angle manifold, up to diffeomorphism, corresponding to the simple polytope obtained by making $l$ corner cuts on a standard simplex.

The varieties discussed above, appearing in work of S. López de Medrano et alia, split after suspending once. Other varieties, given by classical Stiefel manifolds, also stably decompose by work of I. M. James [86], and H. Miller [105]. It is natural to consider the variety given by intersections of these two types of quadratic varieties, as well as examine whether, and how they stably decompose. A result of Cohen and Peterson [47] shows that the stable decompositions for Stiefel varieties generically require many suspensions in order to split.

\section{The COHOMOLOGY OF MOMENT-ANGLE COMPLEXES}

The integral cohomology ring of a moment-angle complex was computed by M. Franz [61] and independently by V. Baskakov, V. Buchstaber and T. Panov [23]. In the latter, the two-disc $D^{2} \subset \mathbb{C}$ is decomposed into three cells: the zero-cell is the point $1 \in D^{2}$, the one-cell, denoted by $T$, is the complement $\partial D^{2} \backslash\{1\}$ and the two-cell, denoted by $D$ is the interior of $D^{2} \subset \mathbb{C}$. Taking products yields a cellular decomposition of $D^{m} \subset \mathbb{C}^{m}$.

Below we summarize the computation, following the development in [36]. The cells of $D^{m}$ are parametrized by pairs of subset $I, J \subset[m]=\{1,2,3, \ldots\}$ satisfying $I \cap J=\varnothing$. The set $J$ parametrizes the $T$-cells and the set $I$ parametrizes the $D$-cells. The remaining positions $[m] \backslash(I \cup J)$ are occupied by 0-cells. The cell corresponding to the pair $J, I$ is denoted $\langle J, I\rangle$. Next, we introduce $K$, a simplicial complex on the vertices $[m]$. In this decomposition, the moment-angle complex $Z\left(K ;\left(D^{2}, S^{1}\right)\right)$ embeds in $D^{m}$ as a cellular subcomplex; the cell $\langle I, J\rangle$ is in $Z\left(K ;\left(D^{2}, S^{1}\right)\right)$ whenever $I$ corresponds to a simplex in $K$.

The cellular cochains $C^{*}\left(Z\left(K ;\left(D^{2}, S^{1}\right)\right)\right)$ have a basis $\langle I, J\rangle^{*}$ dual to the cells. These are bigraded by $\operatorname{deg}\langle I, J\rangle^{*}=(-|J|, 2|I|+2|J|)$. The cellular differential preserves the second grading and so the complex splits as follows:

$$
C^{*}\left(Z\left(K ;\left(D^{2}, S^{1}\right)\right)\right)=\bigoplus_{q=0}^{m} C^{*, 2 q}\left(Z\left(K ;\left(D^{2}, S^{1}\right)\right)\right) .
$$

The bigraded Betti numbers are defined by

$$
b^{-p, 2 q}(K)=\operatorname{rank} H^{-p, 2 q}\left(Z\left(K ;\left(D^{2}, S^{1}\right)\right)\right)
$$

and so the ordinary Betti numbers of the moment-angle complex satisfy

$$
b^{k}(K)=\sum_{-p+2 q=k} b^{-p, 2 q}(K) .
$$

Recall that the integral Stanley-Reisner ring of $K, \mathbb{Z}(K)$, is a polynomial ring on twodimensional generators $v_{i}$, modulo the ideal $I$ generated by monomials corresponding to 
the minimal non-faces of $K$,

$$
\mathbb{Z}(K) \cong \mathbb{Z}\left[v_{1}, \ldots, v_{m}\right] / I
$$

A minimal non-face of $K$ is a sequence of vertices of $K$ which is not a simplex of $K$, but any proper subset is a simplex of $K$.

There is an isomorphism of cochain complexes, [36, Lemma 4.5.1],

$$
g: R^{*}(K) \longrightarrow C^{*}\left(Z\left(K ;\left(D^{2}, S^{1}\right)\right)\right)
$$

where $R^{*}(K)$ is the quotient of a Koszul algebra

$$
\Lambda\left[u_{1}, \ldots, u_{m}\right] \otimes \mathbb{Z}(K) /\left(v_{i}^{2}=u_{i} v_{i}=0,1 \leq i \leq m\right)
$$

with bigrading and differential given by

$$
\operatorname{deg} u_{i}=(-1,2), \quad \operatorname{deg} v_{i}=(0,2), \quad d u_{i}=v_{i}, \quad d v_{i}=0 .
$$

The isomorphism $g$ is given by $u_{J} v_{I} \mapsto\langle I, J\rangle^{*}$. It follows that there is an additive isomorphism

$$
H\left(R^{*}(K)\right) \cong H^{*}\left(Z\left(K ;\left(D^{2}, S^{1}\right)\right)\right) .
$$

The properties of polyhedral products are used then to show that the algebra $R^{*}(K)$ is weakly equivalent to the Koszul algebra as follows;

$$
\Lambda\left[u_{1}, \ldots, u_{m}\right] \otimes \mathbb{Z}(K)=C^{*}\left(Z\left(K ;\left(S^{\infty}, S^{1}\right)\right)\right) \cong C^{*}\left(Z\left(K ;\left(D^{2}, S^{1}\right)\right)\right)=R^{*}(K) .
$$

Furthermore, a cellular diagonal approximation is constructed in [36], which allows for an extension to the multiplicative structure of the cohomology.

Theorem 5.1. [36, Theorem 4.5.4] There are isomorphisms of bigraded algebras

$$
H^{*, *}\left(Z\left(K ;\left(D^{2}, S^{1}\right)\right)\right) \cong \operatorname{Tor}_{\mathbb{Z}\left[v_{1}, \ldots, v_{m}\right]}(\mathbb{Z}(K), \mathbb{Z}) \cong H\left(\Lambda\left[u_{1}, \ldots, u_{m}\right] \otimes \mathbb{Z}(K), d\right)
$$

where the bigrading and differential are as in (5.2). The isomorphisms are natural for simplicial morphisms of $K$, which are assumed to be monomorphisms.

Notice now that the bigraded Betti numbers satisfy

$$
b^{-p, 2 q}(K)=\operatorname{dim} \operatorname{Tor}_{\mathbb{Z}\left[v_{1}, \ldots, v_{m}\right]}^{-p, 2 q}(\mathbb{Z}(K), \mathbb{Z})
$$

and their calculation is simplified by the following theorem due to M. Hochster.

Theorem $5.2([78])$. Let $K_{J}$ denote the full subcomplex of $K$ corresponding to $J \subset[m]$. Then

$$
\operatorname{Tor}_{\mathbb{Z}\left[v_{1}, \ldots, v_{m}\right]}^{-p, 2 q}(\mathbb{Z}(K), \mathbb{Z})=\bigoplus_{J \subset[m],|J|=q} \widetilde{H}^{q-p-1}\left(\left|K_{J}\right| ; \mathbb{Z}\right)
$$

with the convention $\widetilde{H}^{-1}\left(K_{\varnothing} ; \mathbb{Z}\right)=\mathbb{Z}$. 
This leads to a formula for the bigraded Betti numbers in terms of the full subcomplexes

$$
b^{-p, 2 q}(K)=\sum_{J \subset[m],|J|=q} \operatorname{dim} \widetilde{H}^{q-p-1}\left(\left|K_{J}\right| ; \mathbb{Z}\right) .
$$

From Theorem 5.1, (cf. Theorem 8.1), we conclude that the bigraded Betti numbers of the Tor module are the ranks of the contributions of $H^{*}\left(\left|K_{J}\right|\right)$ to the cohomology of $Z\left(K ;\left(D^{2}, S^{1}\right)\right)$. Further computations and properties of the bigraded and multigraded Betti numbers may be found in [8] [97] [133] and [42]. The calculations in [42] are used to verify the Halperin-Carlsson conjecture, ([42, Remark 3]), in the case of free torus actions on moment-angle complexes. This conjecture bounds the ranks of these tori by the sum of the ranks of the cohomology groups.

An analogue of Theorem 5.1 is obtained in [102] for a generalization of moment-angle complexes arising from simplicial posets.

The question whether the cohomology of a moment-angle manifold determines its diffeomorphism type is currently an active area of research, [39].

\section{THE EXPONENTIATION PROPERTy OF POLYHEDRAL PRODUCTS}

A product is defined on CW-pairs by

$$
(X, A) \times(U, V)=\left(X \times U,(A \times U) \cup_{A \times V}(X \times V)\right) .
$$

Let $K$ be a simplicial complex on vertices $[m]$ and $(\underline{X}, \underline{A})$ a family of $\mathrm{CW}$-pairs. For a sequence of positive integers $J=\left(j_{1}, j_{2}, \ldots, j_{m}\right)$, define a new family of pairs $(\underline{Y}, \underline{B})$ by

$$
\left(Y_{i}, B_{i}\right)=\left(X_{i}, A_{i}\right)^{j_{i}}
$$

It turns out that the combinatorics of a polyhedral product can detect this change. Let $K$ be a simplicial complex of dimension $n-1$ on vertices $\left\{v_{1}, v_{2}, \ldots, v_{m}\right\}$. From $K$ and a sequence $J$ as above, a new simplicial complex $K(J)$ exists on $j_{1}+j_{2}+\cdots+j_{m}$ vertices, labelled

$$
v_{11}, v_{12}, \ldots, v_{1 j_{1}}, v_{21}, v_{22}, \ldots, v_{2 j_{2}}, \ldots, v_{m 1}, v_{m 2}, \ldots, v_{m j_{m}} .
$$

It is characterized by the property that

$$
\left\{v_{i_{1} 1}, v_{i_{1} 2}, \ldots, v_{i_{1} j_{1}}, v_{i_{2} 1}, v_{i_{2} 2}, \ldots, v_{i_{2} j_{i_{2}}}, \ldots, v_{i_{k} 1}, v_{i_{k} 2}, \ldots, v_{i_{k} j_{i_{k}}}\right\}
$$

is a minimal non-face of $K(J)$ if and only if $\left\{v_{i_{1}}, v_{i_{2}}, \ldots, v_{i_{k}}\right\}$ is a minimal non-face of $K$. Moreover, all minimal non-faces of $K(J)$ have this form.

An alternative explicit construction of the simplicial complex $K(J)$ may be found in either [15, Construction 2.2] or [119]. The construction of $K(J)$ is known variously in the literature as the simplicial wedge construction or the J-construction.

Next, we adopt the convention of denoting by $Z(K(J) ;(\underline{X}, \underline{A}))$ the polyhedral product determined by the simplicial complex $K(J)$ and the family of pairs obtained from $(\underline{X}, \underline{A})$ 
by repeating each $\left(X_{i}, A_{i}\right), j_{i}$ times in sequence. The main structure theorem is the following.

Theorem 6.1 ([15]). Let $K$ be a simplicial complex with $m$ vertices and let $(\underline{X}, \underline{A})$ denote a family of $C W$-pairs and the family $(\underline{Y}, \underline{B})$ be defined as in (6.1). Then

$$
Z(K(J) ;(\underline{X}, \underline{A}))=Z(K ;(\underline{Y}, \underline{B}))
$$

as subspaces of $X_{1}^{j_{1}} \times X_{2}^{j_{2}} \times \cdots \times X_{m}^{j_{m}}$.

This theorem has applications in toric topology and geometry. In particular, since we have $\left(D^{1}, S^{0}\right)^{2} \cong\left(D^{2}, S^{1}\right)$, it implies that each moment-angle complex $Z\left(K ;\left(D^{2}, S^{1}\right)\right)$ is homeomorphic to a real moment-angle complex $Z\left(K(J) ;\left(D^{1}, S^{0}\right)\right)$ where $J=(2,2, \ldots, 2)$. Three other applications are discussed below.

The cohomology of toric manifolds given by (2.4) and (3.5) has a presentation determined by the combinatorics of $K$ and relations arising from the function $\lambda$. In cases when $z$ in (2.4) has the form $Z\left(K(J) ;\left(D^{2}, S^{1}\right)\right)$, for some $K$ and $J$, Theorem 6.1 can be used to give a simpler presentation of the cohomology of associated toric manifolds which uses the combinatorics of $K$ only. More details can be found in [15].

Remark. A generalization of the Davis-Januszkiewicz construction (2.2) which accommodates the construction here can be found in [14].

An application to topological joins begins by defining a family of CW-pairs

$$
\left.\left(\underline{C\left(*_{J} X\right.}\right), \underline{*_{J} X}\right):=\left\{(C(\underbrace{X_{i} * X_{i} * \cdots * X_{i}}_{j_{i}}), \underbrace{X_{i} * X_{i} * \cdots * X_{i}}_{j_{i}})\right\}_{i=1}^{m} .
$$

for each sequence $J=\left(j_{1}, j_{2}, \ldots, j_{m}\right)$ and family of CW-complexes $\left\{X_{i}\right\}_{i=1}^{m}$. Here, $C(-)$ denotes the unreduced cone. It is shown in [15] that the equivalence $X * X \stackrel{\simeq}{\longrightarrow}(C X \times$ $X) \cup(X \times C X)$ iterates appropriately so that the next theorem follows from Theorem 6.1

Corollary 6.2 ([15]). There is a homeomorphism of polyhedral products,

$$
\left.Z\left(K ;\left(\underline{C\left(*_{J} X\right.}\right), \underline{*_{J} X}\right)\right) \longrightarrow Z(K(J) ;(\underline{C X}, \underline{X})) .
$$

Remark. Notice that in the case $X_{i}=S^{1}$ for all $i$, this corollary yields a homeomorphism

$$
Z\left(K ;\left(\underline{D}^{2 J}, \underline{S}^{2 J-1}\right)\right) \longrightarrow Z\left(K(J) ;\left(D^{2}, S^{1}\right)\right),
$$

where $\left(\underline{D}^{2 J}, \underline{S}^{2 J-1}\right)=\left\{\left(D^{2 j_{i}}, S^{2 j_{i}-1}\right)\right\}_{i=1}^{m}$.

Theorem 6.1 also implies an observation about the action of the Steenrod algebra.

Corollary 6.3 ([15, 12]). There is an isomorphism of ungraded rings

$$
H^{*}\left(Z\left(K ;\left(D^{2}, S^{1}\right)\right) ; \mathbb{Z} / 2\right) \longrightarrow H^{*}\left(Z\left(K(J) ;\left(D^{2}, S^{1}\right)\right) ; \mathbb{Z} / 2\right)
$$


which commutes with the action of the Steenrod algebra.

Remark. The construction of Theorem 6.1 has been generalized by Ayzenberg in [8]; see also [14, Section 6].

\section{Fibrations}

A direct extension of a result of Denham and Suciu [55, Lemma 2.9] yields the next theorem detailing the behaviour of polyhedral products with respect to fibrations.

Theorem 7.1. Let $p_{i}:\left(E_{i}, E_{i}^{\prime}\right) \rightarrow\left(B_{i}, B_{i}^{\prime}\right)$ be a map of pairs, such that $p: E_{i} \rightarrow B_{i}$ and $p \mid E_{i}^{\prime}: E_{i}^{\prime} \rightarrow B_{i}^{\prime}$ are fibrations over path connected $C W$ complexes with fibres $F_{i}$ and $F_{i}^{\prime}$ respectively. Let $K$ be a simplicial complex with $m$ vertices. If either $\underline{B}=\underline{B^{\prime}}$ or $\underline{F}=\underline{F^{\prime}}$, then the following is a fibration:

$$
Z\left(K ;\left(\underline{F}, \underline{F}^{\prime}\right)\right) \rightarrow Z\left(K ;\left(\underline{E}, \underline{E^{\prime}}\right)\right) \rightarrow Z\left(K ;\left(\underline{B}, \underline{B^{\prime}}\right) .\right.
$$

Special cases of this fibration were developed earlier by G. Porter, [117]. He proved that these fibrations exist in the special cases where $(X, A)=(X, *)$ with $A=*$ the base-point, and $K$ is the $q$-skeleton of the full $m$-simplex for $0 \leq q \leq m-1$.

Natural consequences of Theorem 7.1 also arose earlier in the work of T. Ganea [64] and A. Kurosh [92]. G. W. Whitehead had defined a filtration of the product $X_{1} \times \cdots \times X_{m}$, where the $j^{\text {th }}$-filtration is given by the space

$$
W_{j}\left(X_{1}, X_{2}, \ldots, X_{m}\right)=\left\{\left(y_{1}, \ldots, y_{m}\right) \mid y_{i}=*_{i} \in X_{i} \text {, for at least } m-j \text { values of } i\right\} \text {. }
$$

This space is the polyhedral product

$$
W_{j}\left(X_{1}, X_{2}, \ldots, X_{m}\right)=Z\left(\Delta[m-1]_{j-1} ;(\underline{X}, \underline{*})\right),
$$

where $\Delta[m-1]_{q}$ denotes the $q$-skeleton of the $(m-1)$-dimensional simplex. In this case, Porter's result gives an identification of the homotopy theoretic fibre of the inclusion

$$
W_{j}\left(X_{1}, X_{2}, \ldots, X_{m}\right) \subset \prod_{1 \leq i \leq m} X_{i}
$$

which follows directly from the classical path-loop fibration over $X$ and Theorem 7.1 . To see this, we shall extend to the right the fibration of pairs

$$
(\Omega X, \Omega X) \longrightarrow(P X, \Omega X) \stackrel{e_{1}}{\longrightarrow}(X, *),
$$

where $e_{1}$ is the map evaluating the endpoint of a path. Consider again the cone $C Y$ over a space $Y$. There is a map

$$
\begin{aligned}
\kappa: X & \longrightarrow X \times C(P X) \\
x & \mapsto \quad\left(x,\left[0, f_{x}\right]\right)
\end{aligned}
$$

where $f_{x}:[0,1] \rightarrow X$ is the constant path satisfying $f_{x}(t)=x$. The map $\kappa$ is evidently a homotopy equivalence. Consider now $P X$ to be the subspace of $X \times C(P X)$ given by 
pairs $(f(1),[0, f])$ for $f \in P X$. This gives a pair $(X \times C(P X), P X)$. Consequently, we get a fibration of pairs

$$
(C(P X), \Omega X) \longrightarrow(X \times C(P X), P X) \stackrel{\pi_{X} \times e_{1}}{\longrightarrow}(X, X)
$$

for which $\pi_{X}: X \times C(P X) \rightarrow X$ is the natural projection. Applying Theorem 7.1 yields the fibration

$$
Z(K ;(\underline{P X}, \underline{\Omega X})) \longrightarrow Z(K ;(\underline{X \times P X}, \underline{P X})) \stackrel{\pi_{X} \times e_{1}}{\longrightarrow} Z(K ;(\underline{X}, \underline{X})) .
$$

Moreover, the equivalence of pairs $(X, *) \rightarrow(X \times C(P X), P X)$, gives the associated homotopy fibration

$$
Z(K ;(\underline{P X}, \underline{\Omega X})) \longrightarrow Z(K ;(\underline{X}, \underline{*})) \stackrel{\pi_{X} \times e_{1}}{\longrightarrow} Z(K ;(\underline{X}, \underline{X})),
$$

which we record as the next corollary which generalizes Porter's identification.

Corollary 7.2. [11, Corollary 2.32] If all of the $X_{i}$ are path-connected, the homotopy theoretic fibre of the inclusion $Z(K ;(\underline{X}, \underline{*})) \subset Z(K ;(\underline{X}, \underline{X}))$ is $Z(K ;(\underline{P X}, \underline{\Omega X}))$. In particular, the homotopy theoretic fibre of the inclusion $W_{j}\left(X_{1}, X_{2}, \ldots, X_{m}\right) \subset \prod_{i=1}^{m} X_{i}$ is

$$
Z\left(\Delta[m-1]_{j-1} ;(\underline{P X}, \underline{\Omega X})\right) .
$$

For a more general version of this result, see [111, Proposition 5.1]. See also Section 10, and for related information about generalized Whitehead products, [113, Section 7], [71] and [83], where the techniques used involve the fat wedge filtration.

The structure of the fibre $Z\left(\Delta[m-1]_{j-1} ;(\underline{P X}, \underline{\Omega X})\right)$ is given by a second theorem of G. Porter [117]. After one suspension, this result also follows from the next theorem which gives a decomposition of the suspension of $\left.Z\left(\Delta[m-1]_{q} ; \underline{C Y}, \underline{Y}\right)\right)$, for a family of connected, pointed CW-complexes $\left\{Y_{i}, *_{i}\right\}_{i=1}^{m}$. Recall that $\widehat{Y}^{I}$ is $Y_{i_{1}} \wedge \cdots \wedge Y_{i_{k}}$ if $I=\left(i_{1}, \ldots, i_{k}\right)$.

Theorem 7.3. Let $\left\{Y_{i}, *_{i}\right\}_{i=1}^{m}$ be a family of connected, pointed $C W$-complexes. Then there is a homotopy equivalence

$$
\Sigma\left(Z\left(\Delta[m-1]_{q} ;(\underline{C Y}, \underline{Y})\right)\right) \rightarrow \Sigma\left(\bigvee_{q+1<|I| \leq m} \bigvee_{t_{I}}\left(\Sigma^{q+1} \widehat{Y}^{I}\right)\right)
$$

where $t_{I}$ is the binomial coefficient $\left(\begin{array}{c}|I|-1 \\ q+1\end{array}\right)$.

This result is deduced from [11, Theorem 2.19], by a counting argument which uses the fact that here, the full sub-complex $K_{I}$ is an $(|I|-1)$-simplex, and then enumerates the spheres in the $q$-skeleton of the $(|I|-1)$-simplex.

When $q=m-2, Z\left(\Delta[m-1]_{m-2} ;(\underline{X} ; \underline{*})\right)$ is the fat wedge $W_{m-1}\left(X_{1}, X_{2}, \ldots, X_{m}\right)$ and the homotopy fibre is $Z\left(\Delta[m-1]_{m-2} ;(\underline{P X}, \underline{\Omega X})\right)$. In this case, it follows that there is a 
homotopy equivalence

$$
\Sigma\left(Z\left(\Delta[m-1]_{m-2} ;(\underline{P X}, \underline{\Omega X})\right)\right) \rightarrow \Sigma\left(\Omega X_{1} * \ldots * \Omega X_{m}\right) .
$$

T. Ganea [64] identified the homotopy theoretic fibre of the natural inclusion $X \vee Y \rightarrow X \times Y$ as the join $\Omega(X) * \Omega(Y)$ in the case $X$ and $Y$ are path-connected, pointed spaces having the homotopy type of a CW-complex. This example is a special case of the homotopy theoretic fibre for the inclusion of a polyhedral product into a product corresponding to the simplicial complex $K$ given by two discrete points.

\section{Unstable AND STABLE DECOMPOSITIONS OF THE POLYHEDRAL PRODUCT}

A Cartesian product $\left(X_{1} \times \cdots \times X_{m}\right)$ of pointed, connected CW-complexes splits stably by a homotopy equivalence

$$
H: \Sigma\left(X_{1} \times \cdots \times X_{m}\right) \rightarrow \Sigma\left(\bigvee_{I \subseteq[m]} \widehat{X}^{I}\right)
$$

where $I$ runs over all the non-empty sub-sequences of $(1,2, \ldots, m)$. It is natural then to ask if a similar splitting holds for the polyhedral product

$$
Z(K ;(\underline{X}, \underline{A})) \subset X_{1} \times \cdots \times X_{m} .
$$

In other words, does a stable splitting of the polyhedral product exist for simplicial complexes $K \neq \Delta^{m}$ ? Indeed, in the setting of moment-angle complexes, such a splitting was suggested by the cohomology result of V. Baskakov [22] following.

Theorem 8.1. There is an isomorphism of rings

$$
H^{k}\left(Z_{K} ;\left(D^{2}, S^{1}\right)\right) \cong \bigoplus_{I \subset[m]} \widetilde{H}^{k-|I|-1}\left(\left|K_{I}\right|\right),
$$

where the product on the right hand side is that described in Section 11.

This result is deduced from those in Section 5. This decomposition is related to a splitting of the cohomology of the complements of subspace arrangements as follows. A simplicial complex $K$ determines a complex coordinate arrangement of subspaces whose complement is homeomorphic to $Z\left(K ;\left(\mathbb{C}, \mathbb{C}^{*}\right)\right)$. The moment-angle complex $Z\left(K ;\left(D^{2}, S^{1}\right)\right)$ is a strong deformation retract of $Z\left(K ;\left(\mathbb{C}, \mathbb{C}^{*}\right)\right)$, by a result of N. Strickland [123, Proposition 20], (cf. V. Buchstaber and T. Panov [34, Theorem 5.2.5]). In [67], M. Goresky and R. MacPherson derive a decomposition for the cohomology of the complements of subspace arrangements which gives a cohomology splitting analogous to Theorem 8.1. A more direct proof was given by G. Ziegler and R. Živaljević in [135].

The first geometric result in this direction is the unstable splitting due to J. Grbic and S. Theriault, [68, Theorem 1]. They show that when $K$ consists of $m$ discrete points, then 
there is a homotopy equivalence

$$
Z\left(K ;\left(D^{2}, S^{1}\right)\right) \simeq Z\left(K ;\left(\mathbb{C}, \mathbb{C}^{*}\right)\right) \longrightarrow \bigvee_{k=2}^{m}(k-1)\left(\begin{array}{c}
m \\
k
\end{array}\right) S^{k+1}
$$

Their proof uses an inductive argument based on a version of Corollary 7.2. As noted above, the space $Z\left(K ;\left(\mathbb{C}, \mathbb{C}^{*}\right)\right)$ corresponds to the complement of a codimension-two coordinate subspace arrangement. Later in [69], they extended this result to a class of simplicial complexes called shifted, and to simplicial complexes which can be obtained from shifted complexes by certain elementary topological operations.

Definition 8.2. A simplicial complex $K$ is called shifted if there is an ordering of its vertices such that whenever $v$ is a vertex of $\sigma \in K$ and $v^{\prime}<v,(\sigma \backslash v) \cup v^{\prime} \in K$.

Theorem 8.3. [69, Theorem 1.1] If $K$ is a shifted complex, then $Z\left(K ;\left(D^{2}, S^{1}\right)\right)$ is homotopy equivalent to a wedge of spheres.

Following a conjecture made by the authors in [11, this result has been extended in [70] and [79] to the case $Z(K ;(\underline{C X}, \underline{X}))$, as described in Theorem 8.4 below. Indeed, under a condition on $K$, known as dual sequentially Cohen-Macaulay over $\mathbb{Z}$, there is the following improvement over previous results, including those of [73]. (Recognizing that shifted implies dual shifted, arguments from [28] and [29] can be amalgamated to deduce that $K$ shifted implies that it is dual sequentially Cohen-Macaulay over $\mathbb{Z}$.)

Theorem 8.4. [82, Theorems 1.3 and 1.4] If $K$ is dual sequentially Cohen-Macaulay over $\mathbb{Z}$, then

$$
Z(K ;(\underline{C X}, \underline{X})) \simeq \bigvee_{\varnothing \neq I \subset[m]} \Sigma\left|K_{I}\right| \wedge \widehat{X}^{I}
$$

Notice that if $X=S^{1}$, the right hand side is a wedge of spheres.

Example 8.5. A simple example of the splitting in Theorem 8.4 occurs for the simplicial complex consisting of two discrete points, $K=\left\{\varnothing,\left\{v_{1}\right\},\left\{v_{2}\right\}\right\}$. Here $m=2$ and

$$
(\underline{C X}, \underline{X})=\left\{\left(C X_{1}, X_{1}\right),\left(C X_{2}, X_{2}\right)\right\} \text {. }
$$

In this case, 8.2$)$ sees the well known homotopy equivalence $X_{1} * X_{2} \simeq \Sigma\left(X_{1} \wedge X_{2}\right)$ in the form

$$
C X_{1} \times X_{2} \cup_{X_{1} \times X_{2}} X_{1} \times C X_{2} \simeq C X_{1} \vee C X_{2} \vee \Sigma\left(X_{1} \wedge X_{2}\right)
$$

For general $(\underline{X}, \underline{A})$ there is the stable splitting of [10] and [11].

Theorem 8.6. [11, Theorem 2.10] Let $K$ be an abstract simplicial complex on vertices $[m]$. Given $(\underline{X}, \underline{A}))=\left\{\left(X_{i}, A_{i}\right)\right\}_{i=1}^{m}$ where $\left(X_{i}, A_{i}, x_{i}\right)$ are pointed pairs of $C W$-complexes. 
then there is a natural pointed homotopy equivalence

$$
H: \Sigma(Z(K ;(\underline{X}, \underline{A}))) \longrightarrow \Sigma\left(\bigvee_{I \subseteq[m]} \widehat{Z}\left(K_{I} ;(\underline{X}, \underline{A})_{I}\right)\right)
$$

where $(\underline{X}, \underline{A})_{I}$ denotes the restricted family of $C W$-pairs $\left\{\left(X_{i}, A_{i}\right)\right\}_{i \in I}$.

Remark. The spaces $Z(K ;(\underline{X}, \underline{A}))$ generally do not decompose as a wedge before suspending. One example is the simplicial complex $K$ determined by a square, with 4 vertices and 4 edges, for which $Z\left(K ;\left(D^{2}, S^{1}\right)\right)$ is $S^{3} \times S^{3}$.

The homotopy equivalence 8.3 is induced from the following decomposition which is well known, [106, 85].

Theorem 8.7. Let $\left(Y_{i}, y_{i}\right)$ be pointed $C W$-complexes. There is a pointed, natural homotopy equivalence

$$
H: \Sigma\left(Y_{1} \times \cdots \times Y_{m}\right) \rightarrow \Sigma\left(\bigvee_{I \subseteq[m]} \widehat{Y}^{I}\right)
$$

where I runs over all the non-empty subsequences of $(1,2, \ldots, m)$. Furthermore, the map $H$ commutes with colimits.

It is shown in [11] that the map $H$ of Theorem 8.7 induces an equivalence between the diagrams which define the colimits on either side of (8.3), yielding Theorem 8.6.

In many important cases, the spaces which appear on the right hand side of 8.3 can be identified explicitly. Below are a few of the most common of these. We begin with a definition.

Definition 8.8. For $\sigma$ a simplex in $K, \mathrm{lk}_{\sigma}(K)$ the link of $\sigma$ in $K$, is defined to be the simplicial complex for which

$$
\tau \in \operatorname{lk}_{\sigma}(K) \text { if and only if } \tau \cup \sigma \in K .
$$

Theorem 8.9. [1] Let $K$ be an abstract simplicial complex on vertices $[m]$ and $I \subseteq[m]$. If the family $(\underline{X}, \underline{A})$ has the additional property that the inclusion $A_{i} \subset X_{i}$ is null-homotopic for all $i$, then there is a homotopy equivalence

$$
\widehat{Z}\left(K_{I} ;(\underline{X}, \underline{A})_{I}\right) \longrightarrow \bigvee_{\sigma \in K_{I}}\left|\mathrm{lk}_{\sigma}\left(K_{I}\right)\right| * \widehat{D}(\sigma)
$$

which, when combined with (8.3), gives a homotopy equivalence

$$
\Sigma(Z(K ;(\underline{X}, \underline{A}))) \longrightarrow \Sigma\left(\bigvee_{I \subseteq[m]}\left(\bigvee_{\sigma \in K_{I}}\left|\mathrm{lk}_{\sigma}\left(K_{I}\right)\right| * \widehat{D}(\sigma)\right)\right)
$$

(Here, $\widehat{D}(\sigma)$ is defined in Section 1.) The homotopy equivalence (8.4) is a generalization of the wedge lemma of Welker-Ziegler-Živaljević in [131], applied to the smash polyhedral product. 
Next, consider all homology to be taken with coefficients in a field $k$. Define the Poincaré series of a pointed space $X$ of finite type by

$$
P(X, t)=\sum d_{n}(X) t^{n}
$$

where $d_{n}(X)=$ dimension $_{\mathbb{F}} H_{n}(X ; \mathbb{F})$, and the reduced Poincaré series by

$$
\bar{P}(X, t)=-1+P(X, t) .
$$

This series behaves well with respect to wedges and smash products. Hence, (for example), for pairs satisfying the hypotheses of Theorem 8.9 we have the next result.

Lemma 8.10. Assume that homology is taken with field coefficients $\mathbb{F}$ and that path connected pairs of pointed $C W$-complexes $(\underline{X}, \underline{A})$, of finite type, have the property that the inclusion $A_{i} \subset X_{i}$ is null-homotopic for all $i$. Then

$$
\bar{P}(\widehat{Z}(K ;(\underline{X}, \underline{A})), t)=\sum_{\sigma \in K} t \bar{P}\left(\left|\mathrm{lk}_{\sigma}\left(K_{I}\right)\right|, t\right) \bar{P}(\widehat{D}(\sigma), t) .
$$

Notice that if in Theorem 8.9 all the spaces $A_{i}$ are contractible, then the spaces $\widehat{D}(\sigma)$ are contractible unless $I$ represents a simplex $\sigma$ in $K$, that is $K_{I}$ is an $(|I|-1)$-simplex; in this case, $\widehat{D}(\sigma)=\widehat{X}^{I}$ and the next theorem follows.

Theorem 8.11 ([1] $)$. Let $K$ be an abstract simplicial complex with $m$ vertices and $(\underline{X}, \underline{A})$ has the property that all the $A_{i}$ are contractible. Then there is a homotopy equivalence

$$
\widehat{Z}\left(K_{I} ;(\underline{X}, \underline{A})_{I}\right) \longrightarrow \widehat{X}^{I}
$$

yielding a homotopy equivalence

$$
\Sigma(Z(K ;(\underline{X}, \underline{A}))) \longrightarrow \Sigma\left(\bigvee_{I \in K} \widehat{X}^{I}\right) .
$$

The next example is the one for which all the spaces $X_{i}$ are contractible. Now, for each $I \subseteq[m]$, the spaces $\widehat{D}(\sigma)$ in $(8.4)$ are contractible with the possible exception of the case $\sigma=\varnothing$, the empty simplex, which gives $\widehat{D}(\sigma)=\widehat{A}^{I}$. In this case $\left|\mathrm{lk}_{\varnothing}\left(K_{I}\right)\right| \simeq\left|K_{I}\right|$ and (8.4) simplifies to yield the next theorem.

Theorem 8.12. [1] Let $K$ be an abstract simplicial complex with $m$ vertices and suppose that the collection $(\underline{X}, \underline{A})$ has the property that all the $X_{i}$ are contractible. Then there are homotopy equivalences

$$
\widehat{Z}\left(K_{I} ;(\underline{X}, \underline{A})_{I}\right) \longrightarrow\left|K_{I}\right| * \widehat{A}^{I} \longrightarrow \Sigma\left(\left|K_{I}\right| \wedge \widehat{A}^{I}\right)
$$

yielding a homotopy equivalence

$$
\Sigma(Z(K ;(\underline{X}, \underline{A}))) \longrightarrow \Sigma\left(\bigvee_{I \notin K}\left|K_{I}\right| * \widehat{A}^{I}\right) .
$$

The last statement of this theorem follows on observing that if $I \in K$ then $\left|K_{I}\right|$ is contractible. The case $\left(X_{i}, A_{i}, x_{i}\right)=\left(D^{n+1}, S^{n}, *\right)$ for all $i, n \geq 1$, yields the next corollary. 
Corollary 8.13. There are homotopy equivalences

$$
\Sigma\left(Z\left(K ;\left(D^{n+1}, S^{n}\right)\right)\right) \rightarrow \Sigma\left(\bigvee_{I \notin K}\left|K_{I}\right| * S^{n|I|}\right) \rightarrow \bigvee_{I \notin K} \Sigma^{2+n|I|}\left|K_{I}\right|
$$

In the case $n=1$, which corresponds to the case of complements of complex coordinate subspace arrangements, this corollary implies the Goresky-MacPherson cohomological splitting mentioned earlier. To observe this, the reduced cohomology of full sub-complexes of $K$ needs to be related to the reduced homology of certain links in the Alexander dual of $K$. This is done in, for example, [36, Corollary 2.4.6].

\section{Equivariance of the Stable SPlitting AND AN APPlication to Number THEORY}

In the case that all the pairs $\left(X_{i}, A_{i}\right)$ are the same, the automorphism group of $K$, $A u t(K) \subseteq \Sigma_{m}$, acts in a natural way on both sides of the splitting (8.3). Using JamesHopf invariants, A. Al-Raisi [2] shows that there is a stable splitting, as in (8.3), which is equivariant with respect to the action of $A u t(K)$.

Theorem 9.1 ([2]). The adjoint of the stable decomposition (8.3),

$$
\theta_{H}: Z(K ;(X, A)) \longrightarrow J\left(\bigvee_{I \subseteq[m]} \widehat{Z}\left(K_{I} ;(X, A)_{I}\right)\right) \longrightarrow \Omega \Sigma\left(\bigvee_{I \subseteq[m]} \widehat{Z}\left(K_{I} ;(X, A)_{I}\right)\right)
$$

is $\operatorname{Aut}(K)$-equivariant.

When $K=K_{n}$ is the boundary dual of an $n$-gon, (see Section 3), a theorem of Coxeter identifies the real moment-angle manifold $Z\left(K_{n} ;\left(D^{1}, S^{0}\right)\right)$.

Theorem $9.2([49])$. The real moment-angle manifold $Z\left(K_{n} ;\left(D^{1}, S^{0}\right)\right)$ corresponding to an $n$-gon, $n \geq 4$, is an oriented surface of genus $g=1+(n-4) 2^{n-3}$.

Remark. The simplicial complex $K_{n}$ has also been used by S. Das [50], to show directly that the genus of the hypercube graph, the one-skeleton of the $n$-cube, is $1+(n-4) 2^{n-3}$. This is a result due originally to G. Ringel [120] and L. Beineke and F. Harary [24]. The computation is done by embedding equivariantly the hypercube graph into the real moment-angle complex $Z\left(K_{n} ;\left(D^{1}, S^{0}\right)\right)$.

In this simple case, where $\operatorname{Aut}\left(K_{n}\right)=D_{2 n}$, the dihedral group with cyclic subgroup $C_{n}$, Theorem 9.1 has interesting consequences. The real moment-angle manifold is the conduit through which A. Al-Raisi uses Theorem 9.1 to link combinatorics of $K_{n}$ and the action of $C_{n}$ on a choice of basis for the cohomology of a surface of genus $g=1+(n-4) 2^{n-3}$.

Briefly, one first counts the number of orbits of the action of $C_{n}$ by considering, up to rotation, the boundary of an $n$-gon as a 2-colored beaded necklace with $n$ beads. Certain colorings are shown to correspond to generators of $H^{*}\left(Z\left(K_{n} ;\left(D^{1}, S^{0}\right)\right)\right)$. A theorem of 
Burnside gives the number of orbits of the action of $C_{n}$ arising from this choice of basis as

$$
\frac{1}{n}\left(\sum_{d \mid n} \phi(d) 2^{n / d}-(n+1)\right)
$$

where $\phi(d)$ is the Euler phi function.

Next, we think of the sequence of beads as a word made from two letters. A word of length $n$ is called an aperiodic word if it has $n$ distinct rotations. Here a rotation of a word $W=a_{1} a_{2} \ldots a_{n}$ is given by $r(W)=a_{n} a_{1} a_{2} \ldots a_{n-1}$. An equivalence class of an aperiodic word under rotation is called a primitive necklace. A. Al-Raisi shows that the rank of $H_{1}\left(Z\left(K_{n} ;\left(D^{1}, S^{0}\right)\right)\right)$ is given in terms of the number of primitive necklaces. The number of primitive $n$-necklaces on an alphabet of size $k$, denoted by $M(k, n)$, was computed by Moreau in the nineteenth century, [107]

$$
M(k, n)=\frac{1}{n} \sum_{d \mid n} \mu(d) k^{\frac{n}{d}}
$$

where $\mu$ is the Möbius inversion function. The number of orbits of size $d$, where $d \mid n$ and $1<d \leq n$, can be counted with the aid of this theorem. The number of orbits with exactly $n$ elements is given by

$$
\frac{1}{n}\left(\sum_{d \mid n} \mu(d) 2^{n / d}-(n-1)\right) .
$$

The number of orbits of size $d<n$, where $d \mid n$, is given by

$$
\frac{1}{d} \sum_{d_{1} \mid d} \mu\left(d_{1}\right) 2^{d / d_{1}} .
$$

Finally, equating the two different orbit counts, we arrive at Al-Raisi's proof of the cyclotomic identity below.

Theorem 9.3 ([2]). For $n \geq 4$ there is an identity

$$
\frac{1}{n}\left(\sum_{d \mid n} \mu(d) 2^{n / d}-(n-1)\right)+\sum_{\substack{d \mid n \\ 1<d<n}} \frac{1}{d}\left(\sum_{d_{1} \mid d} \mu\left(d_{1}\right) 2^{d / d_{1}}\right)=\frac{1}{n}\left(\sum_{d \mid n} \phi(d) 2^{n / d}-(n+1)\right)
$$

relating the Möbius function $\mu(d)$ and the Euler phi function $\phi(d)$.

The rank of $H_{1}$ above is incidentally given by the rank of certain Lie tensors in a free Lie algebra as first computed by E. Witt [132]. Moreau's formula and Witt's formula agree with one another. This connection between polyhedral products and free Lie algebras is as yet not clearly understood.

These structures together with connections to Hochschild homology as well as new filtrations of classifying spaces will appear in [3]. Additional interesting work subsequent to [2], but from a different point of view, was developed by X. Fu and J. Grbić. They 
construct a sequence of simplicial complexes $\left\{L_{m}\right\}$ with each $L_{m}$ obtained from the $m$-cube by a vertex cut, [63, Construction 4.4] and verify a form of $\Sigma_{m}$-representation stabilty in characteristic zero, for the homology of $Z\left(L_{m} ;(X, A)\right)$.

In a recent preprint, S. Cho, S. Choi and S. Kaji [44], also address the representations afforded by this action. They compute some actions and the associated representations on the homology of $Z\left(K_{n} ;\left(D^{1}, S^{0}\right)\right)$ in characteristic zero.

\section{The CASE THAT $A_{i}=*$ FOR ALL $i$}

The stable structure of the polyhedral product $Z(K ;(\underline{X}, \underline{*}))$ is given by Theorem 8.11 . The importance of this particular case of the polyhedral product first came to light in the development of toric topology with the following observation.

Theorem 10.1. [36, Theorem 4.3.2]. The inclusion $i: Z\left(K ;\left(\mathbb{C P}^{\infty}, *\right)\right) \longrightarrow\left(\mathbb{C P}^{\infty}\right)^{m}$ factors into a composition of a homotopy equivalence

$$
Z\left(K ;\left(\mathbb{C P}^{\infty}, *\right)\right) \longrightarrow E T^{m} \times_{T^{m}} Z\left(K ;\left(D^{2}, S^{1}\right)\right)
$$

and the fibration $E T^{m} \times_{T^{m}} Z\left(K ;\left(D^{2}, S^{1}\right)\right) \longrightarrow B T^{m}=\left(\mathbb{C P}^{\infty}\right)^{m}$. The action of the torus $T^{m}$ here is that of (3.1).

This gives one of the most important fibrations in this subject;

$$
Z\left(K ;\left(D^{2}, S^{1}\right)\right) \stackrel{\widetilde{\omega}}{\longrightarrow} Z\left(K ;\left(\mathbb{C P}^{\infty}, *\right)\right) \longrightarrow\left(\mathbb{C P}^{\infty}\right)^{m} .
$$

The cohomology of the right-hand side of (10.1) was determined by geometers in the context of toric geometry, [31, 27]. In the setting of toric topology, it appears in the paper of M. Davis and T. Januszkiewicz, [52, Theorem 4.8]. This calculation, together with the theorem, implies the following result in algebraic combinatorics, for which independent proofs exist.

Corollary 10.2 ([34, 11]). Let $K$ be a simplicial complex. Then

$$
H^{*}\left(Z\left(K ;\left(\mathbb{C P}^{\infty}, *\right)\right) ; \mathbb{Z}\right) \cong \mathbb{Z}(K)
$$

the Stanley-Reisner ring of $K$.

Recall that Theorem 5.1 tells us that $\mathbb{Z}(K)$, determines the cohomology of the momentangle complex $Z\left(K ;\left(D^{2}, S^{1}\right)\right)$.

An analogue of Corollary 10.2 holds over any field $k$ for the ring $k(K)$. Somewhat surprisingly, $k(K)$ determines $K$ by a theorem of W. Bruns and J. Gubeladze, [33].

Theorem 10.3 ([33]). Let $K_{1}$ and $K_{2}$ be simplicial complexes satisfying $k\left(K_{1}\right) \cong k\left(K_{2}\right)$ as $k$-algebras. Then $K_{1}$ and $K_{2}$ are isomorphic as simplicial complexes.

Over any ring $k$ there is also the exterior Stanley-Reisner ring over $k$, which we denote by $\Lambda_{k}(K)$. It is the quotient of a graded exterior algebra on $m$ one-dimensional generators, 
by the same two-sided ideal as in (5.1). In a manner entirely analogous to the proof of Corollary 10.2 from Theorem 8.11, we have

$$
\Lambda_{k}(K) \cong H^{*}\left(Z\left(K ;\left(S^{1}, *\right)\right) ; k\right) .
$$

Using the theory of algebraic groups, C. Stretch, (unpublished), asserts that (10.3) determines $K$ up to isomorphism. In order to compare the Stanley-Reisner ring to 10.3 from a geometric perspective, it is convenient to work over $k=\mathbb{Z}_{2}$ and to use the ungraded isomorphism of rings, (Theorem 8.11, again),

$$
\Lambda_{\mathbb{Z}_{2}}(K) \cong H^{*}\left(Z\left(K ;\left(S^{2}, *\right)\right) ; \mathbb{Z}_{2}\right), \quad \text { (as ungraded rings) } .
$$

The fibrations associated to (7.1) or 14.3), give a commutative diagram

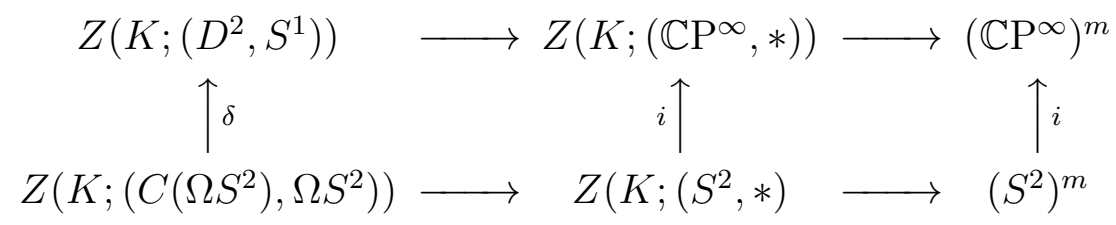

where the right hand vertical maps are determined by the inclusion $S^{2} \stackrel{i}{\rightarrow} \mathbb{C P}^{\infty}$. In cohomology with $\mathbb{Z}_{2}$ coefficients, the middle map determines the natural map from the $\operatorname{ring} \mathbb{Z}_{2}(K)$ to the algebra $\Lambda_{\mathbb{Z}_{2}}(K)$ of $(10.4)$. The map

$$
\Omega S^{2} \stackrel{\Omega i}{\longrightarrow} \Omega \mathbb{C P}^{\infty} \simeq \Omega B S^{1} \simeq S^{1}
$$

induces the vertical map $\delta$, and is given by the projection $\Omega S^{2} \simeq S^{1} \times \Omega S^{3} \longrightarrow S^{1}$, since this detects the generator of $\pi_{1}\left(\Omega S^{2}\right)$.

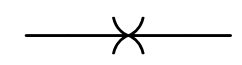

It follows from (10.1) that the Borel construction is a CW-subcomplex of $\left(\mathbb{C P}^{\infty}\right)^{m}$. In particular it has cells in even degree only. In the literature the space $Z\left(K ;\left(\mathbb{C P}^{\infty}, *\right)\right)$ is often denoted by $D J(K)$ or $D J_{K}$ and is called the Davis-Januszkiewicz space.

The integral and rational formality of the Davis-Januszkiewicz spaces is studied by M. Franz in [62], and further by D. Notbohm and N. Ray in [108], using model category theory. They show that the rationalization of $D J(K)$ is unique for a class of simplicial complexes $K$.

Remark. Under suitable freeness conditions, there is an analogue, $H^{*}(Z(K ;(X, *)) ; \mathbb{Z})$, of the Stanley-Reisner ring for any space $X$ and simplicial complex $K$, which specializes to the classsical Stanley-Reisner ring in case $X$ is $\mathbb{C P}^{\infty}$. The result follows from Theorem 8.11, and for more details, [10, Theorem 2.35]. Example 11.4 in Section 11 gives a different perspective and a more concise description of the ring under discussion here. It is in fact the case that all monomial ideal rings can be realized as cohomology rings of spaces related to certain polyhedral products, [13]. 
Unstable phenomena in the homotopy of the left-hand side of 10.1 has been investigated by D. Allen and J. La Luz [94, 4] using the Unstable Novikov Spectral Sequence. In particular, their study of the derived functors of the indecomposables of the StanleyReisner ring, leads to an interesting and mysterious invariant of simplicial complexes.

Next, we consider the relationship between the space $Z\left(K ;\left(S^{1}, *\right)\right)$ and the right-angled Artin group determined by $K$.

Definition 10.4. Given a simplicial graph $\Gamma$ with vertex set $S$ and a family of groups $\left\{G_{s}\right\}_{s \in S}$, their graph product $\prod_{\Gamma} G_{s}$ is the quotient of the free product of the groups $G_{s}$ by the relations that elements of $G_{s}$ and $G_{t}$ commute whenever $\{s, t\}$ is an edge of $\Gamma$. In the case that $G_{i}=\mathbb{Z}$ and $\Gamma=S K$, the one-skeleton of $K$, the graph product is called the right-angled Artin group, $R A_{K}$, corresponding to $K$. When $G_{i}=\mathbb{Z}_{2}$, the graph product is called the right-angled Coxeter group, $R C_{K}$. A simplicial complex for which every collection of pairwise adjacent vertices spans a simplex, is called a flag complex.

Theorem $10.5\left(\left[32\right.\right.$, 90, 53, 43, 114]). The space $Z\left(K ;\left(S^{1}, *\right)\right)$ is aspherical if and only if $K$ is a flag complex, in which case, $\pi_{1}\left(Z\left(K ;\left(S^{1}, *\right)\right)\right)=R A_{K}$.

Related results, [51, 114], identify $\pi_{1}\left(Z\left(K ;\left(\mathbb{R P} \mathrm{P}^{\infty}, *\right)\right)\right)$ as a right-angled Coxeter groups $R C_{K}$. From this follows the fact that $\pi_{1}\left(Z\left(K ;\left(D^{1}, S^{0}\right)\right)\right)=\left[R C_{K}, R C_{K}\right]$, the commutator subgroup.

A general result in this direction is due to M. Stafa. A proof is sketched below using a recent result about the homotopy type of $\Omega Z(K ;(\underline{X}, \underline{*}))$ due to T. Panov and $\mathrm{S}$. Theriault, [113.

Theorem 10.6 ([122]). Let $G_{1}, G_{2}, \ldots, G_{m}$ be non-trivial discrete groups and $K$ be a simplicial complex with $m$ vertices. Then $Z(K ;(\underline{B G}, \underline{*}))$ is an Eilenberg-Mac Lane space if and only if $K$ is a flag complex. Equivalently, $Z(K ;(\underline{E G}, \underline{G}))$ is an Eilenberg-Mac Lane space if and only if $K$ is a flag complex.

Proof. (Sketch.) For the simplicial complex $V_{K}$, consisting of the vertices of $K$, there is a natural map

$$
X_{1} \vee X_{2} \vee \cdots \vee X_{m} \stackrel{\simeq}{\rightarrow} Z\left(V_{K} ;(\underline{X}, \underline{*})\right) \stackrel{i}{\rightarrow} Z(K ;(\underline{X}, \underline{*}))
$$

where the first equivalence is that of 8.5 and the second map is induced by the inclusion $V_{K} \hookrightarrow K$. For a flag complex $K, \mathrm{~T}$. Panov and S. Theriault show in [113] that $\Omega i$ has a right homotopy inverse

$$
\Omega\left(X_{1} \vee X_{2} \vee \cdots \vee X_{m}\right) \longleftarrow \Omega Z(K ;(\underline{X}, \underline{*})) .
$$

so that $\Omega Z(K ;(\underline{X}, \underline{*}))$ is a retract of $\Omega\left(X_{1} \vee X_{2} \vee \cdots \vee X_{m}\right)$. 
When $X_{i}=B G_{i}$ it is known that $B G_{1} \vee B G_{2} \vee \cdots \vee B G_{m} \simeq B\left(G_{1} * G_{2} * \cdots * G_{m}\right)$, and so for $K$ a flag complex, it follows immediately that $Z(K ;(\underline{B G}, \underline{*}))$ is an Eilenberg-Mac Lane space.

If $K$ is not a flag complex, then $\partial \Delta^{n}$, the boundary of an $n$-simplex, is a full subcomplex of $K$ for some $n>1$. In this case, [55, Proposition 3.3.1] implies that $Z\left(\partial \Delta^{n} ;(\underline{B G}, \underline{*})\right)$ splits off from $Z(K ;(\underline{B G}, \underline{*}))$. Hence it suffices to show that $Z\left(\partial \Delta^{n} ;(\underline{B G}, \underline{*})\right)$ has nontrivial higher homotopy groups. For the simplicial complex $\partial \Delta^{n}$, the fibration of Theorem 7.1 takes the form

$$
\left.Z\left(\partial \Delta^{n} ;(\underline{E G}, \underline{G})\right) \longrightarrow Z\left(\partial \Delta^{n} ;(\underline{B G}), \underline{*}\right)\right) \longrightarrow B G_{1} \times B G_{2} \times \cdots \times B G_{n+1} .
$$

Next, since $\partial \Delta^{n}$ is a shifted simplicial complex, (Definition 8.2), and $E G$ is contractible, Theorem 8.4 gives

$$
Z\left(\partial \Delta^{n} ;(\underline{E G}, \underline{G})\right) \simeq S^{n} \wedge G_{1} \wedge G_{2} \wedge \cdots \wedge G_{n+1} .
$$

Since the groups $G_{i}$ are discrete for all $i, Z\left(\partial \Delta^{n} ;(\underline{E G}, \underline{G})\right)$ becomes a wedge of $n$-spheres. The assumption $n>1$, the Hilton-Milnor theorem, and the homotopy sequence of (10.7) imply that $Z\left(\partial \Delta^{n} ;(\underline{B G}, \underline{*})\right)$ has non-trivial higher homotopy groups which completes the proof.

M. Stafa [121] also uses polyhedral products to construct monodromy representations of a finite product of discrete groups into outer automorphisms of free groups. We close this section by noting that the classifying spaces of certain groups derived from right-angled Coxeter groups through quandles, are described in terms of polyhedral products, with $X_{i} \neq *$ and $A_{i} \neq *$, by D. Kishimoto in [91].

\section{The cohomology of polyhedral products and a speCtral sequence}

The right adjoint of the stable splitting Theorem 8.6 induces the product structure in cohomology of a polyhedral product by observing the consequences of suspending the diagonal map. We begin by defining

$$
\mathcal{H}^{q}(K ;(\underline{X}, \underline{A}))=\bigoplus_{I \subseteq[m]} H^{q}\left(\widehat{Z}\left(K_{I} ;(\underline{X}, \underline{A})_{I}\right) .\right.
$$

Next, we impose an algebra structure on $\mathcal{H}^{q}(K ;(\underline{X}, \underline{A}))$. In [12, Section 3], it is shown that whenever $I=J \cup L$, there are well defined maps, partial diagonals, 


$$
\widehat{\Delta}_{I}^{J, L}: \widehat{Z}\left(K_{I} ;(\underline{X}, \underline{A})_{I}\right) \longrightarrow \widehat{Z}\left(K_{J} ;(\underline{X}, \underline{A})_{J}\right) \wedge \widehat{Z}\left(K_{L} ;(\underline{X}, \underline{A})_{L}\right)
$$

making the diagram below commute

$$
\begin{array}{ccc}
Z(K ;(\underline{X}, \underline{A})) \stackrel{\Delta_{K}}{\longrightarrow} & Z(K ;(\underline{X}, \underline{A})) \wedge Z(K ;(\underline{X}, \underline{A})) \\
\downarrow \widehat{\Pi}_{I} & \widehat{\Pi}_{J} \wedge \widehat{\Pi}_{L} \downarrow \\
\widehat{Z}\left(K_{I} ;(\underline{X}, \underline{A})_{I}\right) \stackrel{\widehat{\Delta}_{I}^{J, L}}{\longrightarrow} \widehat{Z}\left(K_{J} ;(\underline{X}, \underline{A})_{J}\right) \wedge \widehat{Z}\left(K_{L} ;(\underline{X}, \underline{A})_{L}\right),
\end{array}
$$

where $\Delta_{K}$ is the reduced diagonal map. The maps $\Pi_{I}, \Pi_{J}$ and $\Pi_{L}$ are induced by the appropriate projection maps

$$
\Pi_{S}: Y^{[m]}=Y_{1} \times Y_{2} \times \cdots \times Y_{m} \longrightarrow Y_{s_{1}} \wedge Y_{s_{2}} \wedge \cdots \wedge Y_{s_{k}}
$$

where $S=\left\{s_{1}, s_{2}, \ldots, s_{k}\right\}$ corresponds in turn to $I, J$ and $L$. This allows us to define a product on $\mathcal{H}^{q}(K ;(\underline{X}, \underline{A}))$ as follows. Given cohomology classes $u \in H^{p}\left(\widehat{Z}\left(K_{J} ;(\underline{X}, \underline{A})_{J}\right)\right)$ and $v \in H^{q}\left(\widehat{Z}\left(K_{L} ;(\underline{X}, \underline{A})_{L}\right)\right)$, define

$$
u * v=\left(\widehat{\Delta}_{I}^{J, L}\right)^{*}(u \otimes v) \in H^{p+q}\left(\widehat{Z}\left(K_{I}\right)\right) .
$$

The element $u * v \in H^{p+q}\left(\widehat{Z}\left(K_{I}\right)\right)$ is called the *-product of $u$ and $v$. Moreover, the commutativity of diagram 11.1 gives

$$
\widehat{\Pi}_{I}^{*}(u * v)=\widehat{\Pi}_{J}^{*}(u) \cup \widehat{\Pi}_{L}^{*}(v)
$$

where $\cup$ is the cup product for the $\mathrm{CW}$-complex $Z(K ;(\underline{X}, \underline{A}))$. So, the $*$-product gives $\mathcal{H}^{q}(K ;(\underline{X}, \underline{A}))$ a ring structure. Next, consider the map

$$
\eta=\bigoplus_{I \subseteq[m]} \Pi_{I}^{*}: \mathcal{H}^{*}(K ;(\underline{X}, \underline{A})) \longrightarrow H^{*}(Z(K ;(\underline{X}, \underline{A})))
$$

which the stable splitting (8.3) ensures is an additive isomorphism. We can now state the main theorem of this section.

Theorem $11.1([12])$. Let $K$ be an abstract simplicial complex with $m$ vertices and assume that $(\underline{X}, \underline{A})=\left\{\left(X_{i}, A_{i}, x_{i}\right)\right\}_{i=1}^{m}$ is a family of based $C W$-pairs. Then

$$
\eta: \mathcal{H}^{*}(K ;(\underline{X}, \underline{A})) \rightarrow H^{*}(Z(K ;(\underline{X}, \underline{A})))
$$

is a ring isomorphism.

It follows that the $*$-product gives $\mathcal{H}^{q}(K ;(\underline{X}, \underline{A}))$ an algebra structure. More details can be found in [12, Section 3]. This theorem generalizes the result of V. Baskakov [22] who proved it for the case of moment-angle complexes, beginning with the cohomological splitting of Theorem 8.1 .

Remark. As discussed in [12], various corollaries follow from Theorem 11.1. Among these are to be found: 
(1) If $(\underline{X}, \underline{A})$ consists of suspension pairs and $J \cap L \neq \varnothing$, then $u * v=0$ for all $u \in H^{p}\left(\widehat{Z}\left(K_{J} ;(\underline{X}, \underline{A})_{J}\right)\right)$ and $v \in H^{q}\left(\widehat{Z}\left(K_{L} ;(\underline{X}, \underline{A})_{L}\right)\right)$.

(2) Whenever $(\underline{C X}, \underline{X})=\left\{\left(C X_{i}, X_{i}, x_{i}\right)\right\}_{i=1}^{m}$ is such that any finite product of the $X_{i}$ with the spaces $Z\left(K_{I} ;\left(D^{1}, S^{0}\right)\right)$ satisfies the strong form of the Künneth theorem, the cup product structure for the cohomology algebra $H^{*}(Z(K ;(\underline{C X}, \underline{X})))$ is a functor of the cohomology algebras of $X$, and $\left.Z\left(K_{I} ;\left(D^{1}, S^{0}\right)\right)\right)$ for all $I$. Real moment-angle complexes $Z\left(L ;\left(D^{1}, S^{0}\right)\right)$ ) have been studied extensively in the work of A. Suciu and A. Trevisan [124], A. Trevisan [127], L. Cai [40] and L. Cai and S. Choi [41].

We turn our attention now to the computation of the cohomology groups of a polyhedral product $H^{*}(Z(K ;(\underline{X}, \underline{A})))$. For a suitable collection of pairs of spaces $(\underline{X}, \underline{A})$, the cohomology of the polyhedral product $Z(K ;(\underline{X}, \underline{A}))$ is computed using a spectral sequence by the authors in [16]. A computation using different methods by Q. Zheng can be found in [134]. The family of pairs $(\underline{X}, \underline{A})$ amenable to a cohomology calculation of $Z(K ;(\underline{X}, \underline{A}))$ satisfy a strong freeness condition, [16, Definition 2.2].

Definition 11.2. The homology of $(\underline{X}, \underline{A})$ with coefficients in a ring $k$, is said to be strongly free if the long exact sequence

$$
\stackrel{\delta}{\rightarrow} \widetilde{H}^{*}\left(X_{i} / A_{i}\right) \stackrel{\ell}{\rightarrow} \widetilde{H}^{*}\left(X_{i}\right) \stackrel{\iota}{\rightarrow} \widetilde{H}^{*}\left(A_{i}\right) \stackrel{\delta}{\rightarrow} \widetilde{H}^{*+1}\left(X_{i} / A_{i}\right) \rightarrow
$$

satisfies the condition that there exist isomorphisms

(1) $\widetilde{H}^{*}\left(A_{i}\right) \cong E_{i} \oplus B_{i}$.

(2) $\widetilde{H}^{*}\left(X_{i}\right) \cong B_{i} \oplus C_{i}$, where $B_{i} \stackrel{\iota}{\simeq} B_{i},\left.\quad \iota\right|_{C_{i}}=0$

(3) $\widetilde{H}^{*}\left(X_{i} / A_{i}\right) \cong C_{i} \oplus W_{i}$, where $C_{i} \stackrel{\ell}{\stackrel{\ell}{\longrightarrow}} C_{i},\left.\quad \ell\right|_{W_{i}}=0, \quad E_{i} \stackrel{\delta}{\stackrel{\varrho}{\longrightarrow}} W_{i}$

for graded modules $E_{i}, B_{i}, C_{i}$ and $W_{i}$ of finite type and free over $k$.

In particular, for finite dimensional complexes, this happens when coefficients are taken in a field. In order to describe the cohomology, more notation is required. For a simplicial complex $K$ with vertices in $[m]=\{1,2,3, \ldots, m\}$, and a subset $J=\left\{j_{1}, \cdots, j_{r}\right\} \subset[m]$, set $E^{J}=E_{j_{1}} \otimes \cdots \otimes E_{j_{r}}$. Let $K_{J}$ be the full subcomplex with vertices in $J$. For $I=\left\{i_{1}, \cdots, i_{t}\right\} \subset[m]$, and for $\sigma \subset I$, set $Y^{I, \sigma}=Y_{1} \otimes \cdots \otimes Y_{t}$ where

$$
Y_{j}= \begin{cases}C_{i_{j}} & \text { if } i_{j} \in \sigma \\ B_{i_{j}} & \text { if } i_{j} \notin \sigma .\end{cases}
$$

and for $I=\varnothing$, set $Y^{\varnothing, \varnothing}=k$. 
Theorem 11.3. If $(\underline{X}, \underline{A})$ satisfies the strong freeness condition there is a direct sum decomposition of the cohomology group

$$
\widetilde{H}^{*}(\widehat{Z}(K ;(\underline{X}, \underline{A})))=\bigoplus_{\substack{\sigma \subset I, \sigma \in K \\ I \cup J=[m], I \cap J=\varnothing}} E^{J} \otimes \widetilde{H}^{*}\left(\Sigma\left|\mathrm{lk}_{\sigma}\left(K_{J}\right)\right|\right) \otimes Y^{I, \sigma}
$$

with the convention that $\widetilde{H}^{*}(\Sigma \varnothing)=k$. (Recall that $\operatorname{lk}_{\sigma}\left(K_{J}\right)$ is defined in Definition 8.8.)

Combined with the splitting Theorem 8.6 in Section 8, this theorem gives a complete description of the cohomology of the topological spaces $Z(K ;(\underline{X}, \underline{A}))$ with appropriate coefficients. Moreover, the theorem generalizes to any multiplicative cohomology theory $h^{*}$ for which $(\underline{X}, \underline{A})$ satisfies the natural formulation of the strong freeness condition for $h^{*}$. More information about the cohomology ring may be found in [16] and [134]. The use of the methods developed in [16] is illustrated next with a few examples, each having a different flavor.

Example 11.4. In the particular case that $H^{*}$ is cohomology with coefficients in a field $k$, (or over $\mathbb{Z}$, under suitable freeness conditions), and the map $H^{*}\left(X_{i}\right) \longrightarrow H^{*}\left(A_{i}\right)$ is surjective for all $i=1,2, \ldots, m$, the spectral sequence constructed in [16] collapses by [16, Proposition 3.8], and allows for a more concise statement of Theorem 11.3. The assumption allows us to consider $H^{*}\left(X_{i} / A_{i}\right)$ as a subring of $H^{*}\left(X_{i}\right)$ and then [16, Corollary 3.9] gives an isomorphism of rings

$$
H^{*}(Z(K ;(\underline{X}, \underline{A}))) \cong H^{*}\left(X_{1}\right) \otimes H^{*}\left(X_{2}\right) \otimes \cdots \otimes H^{*}\left(X_{m}\right) / I
$$

where $I$ is the ideal generated by $H^{*}\left(X_{j_{1}} / A_{j_{1}}\right) \otimes H^{*}\left(X_{j_{2}} / A_{j_{2}}\right) \otimes \cdots \otimes H^{*}\left(X_{j_{t}} / A_{j_{t}}\right)$ with $\left(j_{1}, j_{2}, \ldots, j_{t}\right)$ not spanning a simplex in $K$.

Remark. Notice that this generalizes Corollary 10.2.

$$
H^{*}\left(Z\left(K ;\left(\mathbb{C} P^{\infty}, *\right) ; k\right)\right) \cong k(K)
$$

where $k(K)$ denotes the Stanley-Reisner ring of the simplicial complex $K$.

Example 11.5. Another tractable example is the important case

$$
(\underline{X}, \underline{A})=(\underline{C A}, \underline{A})
$$

which includes the example of moment-angle complexes. In this case, with $H^{*}\left(A_{i}\right)$ free, the modules in the strong freeness condition of Theorem 11.3 are

$$
E_{i}=\widetilde{H}^{*}\left(A_{i}\right), \quad B_{i}=0, \quad C_{i}=0, \text { and } W_{i}=\widetilde{H}^{*}\left(\Sigma A_{i}\right) .
$$

The modules $Y^{I, \sigma}$ of Theorem 11.3, take a particularly simple form: $Y^{I, \sigma}=0$ if $I \neq \varnothing$ and is equal to $k$ if $I=\varnothing$, (see (11.3), item (1)). As a consequence, the only links that 
appear in Theorem 11.3 are $\mathrm{lk}_{\varnothing}(K)=K$ and we have

$$
\widetilde{H}^{*}\left(\widehat{Z}(K ;(\underline{C A}, \underline{A}))=\widetilde{H}^{*}(\Sigma|K|) \otimes \widetilde{H}^{*}\left(A_{1}\right) \otimes \cdots \otimes \widetilde{H}^{*}\left(A_{m}\right) .\right.
$$

This result agrees with the one given by the wedge lemma [131] as described in [11]. Finally, the splitting theorem [11, Theorem 2.10] gives the cohomology of the polyhedral product as

$$
\begin{aligned}
H^{*}(Z(K ;(\underline{C A}, \underline{A}))) & =\bigoplus_{I \subset[m]} H^{*}\left(\Sigma\left|K_{I}\right|\right) \otimes \widetilde{H}^{*}\left(A_{i_{1}}\right) \otimes \cdots \otimes \widetilde{H}^{*}\left(A_{i_{t}}\right) \\
& \subset H^{*}\left(Z\left(K ;\left(D^{1}, S^{0}\right)\right)\right) \otimes H^{*}\left(A_{1}\right) \otimes \cdots \otimes H^{*}\left(A_{m}\right) .
\end{aligned}
$$

There is now an evident product on $H^{*}(Z(K ;(\underline{C A}, \underline{A})))$ induced by coordinate-wise multiplication and by the product in $H^{*}\left(Z\left(K ;\left(D^{1}, S^{0}\right)\right)\right)$, which is the case corresponding to $A_{i}=S^{0}$. The latter is described in [40]. In [16] it is shown, using the results of [12], that this is indeed the ring structure in $H^{*}(Z(K ;(\underline{C A}, \underline{A})))$.

Remark. The ring structure for general $(\underline{X}, \underline{A})$ satisfying the strong freeness condition may be found in [12], [16] and [134].

The next example is of a different nature.

Example 11.6. Consider a CW-pair $(X, A)$ with cohomology satisfying

$$
H^{*}(X)=\mathbb{Z}\left\{b_{4}, c_{6}\right\} \quad \text { and } \quad H^{*}(A)=\mathbb{Z}\left\{e_{2}, b_{4}\right\}
$$

where the dimensions of the classes are given by the subscripts. A trivial example of $(\underline{X}, \underline{A})$ is given by wedges of spheres in the appropriate dimensions. A more interesting example is obtained from the mapping cylinder of the composite map

$$
f: \mathbb{C} P^{2} \hookrightarrow \mathbb{C} P^{3} \rightarrow \mathbb{C} P^{3} / \mathbb{C} P^{1} .
$$

We denote the mapping cylinder of $(11.6)$ by $M_{f}$ and consider the pair $\left(M_{f}, \mathbb{C} P^{2}\right)$, which satisfies the cohomology condition above.

Remark. Notice here that Theorem 11.3 will give the same cohomology for $\widehat{Z}(K ;(\underline{X}, \underline{A}))$ whether we realize condition (11.5) with a pair made from appropriate wedges of spheres, or from the projective spaces above. Given $K$, the cohomology of $\widehat{Z}(K ;(\underline{X}, \underline{A}))$ depends on the modules $E_{i}, B_{i}$ and $C_{i}$ only.

For the example at hand, let $K$ be the simplicial complex with 3 vertices and edges $\{1,3\},\{1,2\}$. The cases in this example are indexed by the $I$ in Theorem 11.3 starting with $I=\varnothing$ and building up to $I=\{1,2,3\}$. For each $I$ there are the sub-cases indexed by the simplices $\sigma \subset I$. Theorem 11.3 reduces the calculation to bookkeeping, as it does with every example, but the bookkeeping can become quite complicated. 
(1) $I=\varnothing, \sigma=\varnothing$.

Here $J=\{1,2,3\}$ and $\left|\mathrm{lk}_{\varnothing}\left(K_{J}\right)\right|=|K|$ is contractible. So, there is no contribution to the Poincaré series for $H^{*}(\widehat{Z}(K ;(\underline{X}, \underline{A})))$ in this case.

(2) $I=\{1\}, \sigma=\varnothing$.

Now, $J=\{2,3\}$, so $E^{J}$ contributes $\left(t^{2}\right)^{2}, Y^{I, \varnothing}=b_{4}$ which gives a $t^{4}$, and $\left|\mathrm{lk}_{\varnothing}\left(K_{J}\right)\right|=|\{\{2\},\{3\}\}|=S^{0}$. So for this case, Theorem 11.3 specifies a total contribution of $t^{9}$ to the Poincaré series for $H^{*}(\widehat{Z}(K ;(\underline{X}, \underline{A})))$.

(3) $I=\{1\}, \sigma=\{1\}$.

Again, $J=\{2,3\}$, so $E^{J}$ contributes $\left(t^{2}\right)^{2}, Y^{I,\{1\}}=c_{6}$ which gives a $t^{6}$, and $\left|\operatorname{lk}_{\{1\}}\left(K_{J}\right)\right|=|\{\{2\},\{3\}\}|=S^{0}$. So for this case, Theorem 11.3 specifies a total contribution of $t^{11}$ to the Poincaré series for $H^{*}(\widehat{Z}(K ;(\underline{X}, \underline{A})))$.

Continuing in this way, we arrive at the (reduced) Poincaré series

$$
\bar{P}\left(H^{*}(\widehat{Z}(K ;(\underline{X}, \underline{A})))\right)=t^{9}+t^{11}+3 t^{12}+5 t^{14}+2 t^{16} .
$$

Remark. This illustrative example lends itself to direct calculation. For $K$ as above, it follows directly from the definition that

$$
\widehat{Z}(K ;(X, A))=X \wedge((X \wedge A) \cup(A \wedge X)) .
$$

The Künneth theorem now reduces the calculation to $H^{*}((X \wedge A) \cup(A \wedge X))$, which is direct for the pair $(X, A)$ in Example 11.6 by the Mayer-Vietoris sequence.

\section{A geOMETRIC APPROACH TO THE COHOMOLOGY OF POLYHEDRAL PRODUCTS}

In the forthcoming paper [17], the authors show that for certain pairs $(\underline{X}, \underline{A})$, called wedge decomposable, the algebraic decomposition given by Theorem 11.3 is a consequence of an underlying geometric splitting. Moreover, the consequences of this observation extend to more general based $C W$-pairs. (The results of this section are from the authors' unpublished preprint from 2014, which in turn originated from an earlier preprint from 2010, and is currently being revised.)

Definition 12.1. Based CW-pairs of the form

$$
(\underline{X}, \underline{A})=(\underline{B \vee C}, \underline{B \vee E})
$$

so that, for all $i,\left(X_{i}, A_{i}\right)=\left(B_{i} \vee C_{i}, B_{i} \vee E_{i}\right)$, where $E_{i} \hookrightarrow C_{i}$ is a null homotopic inclusion, are called wedge decomposable.

For such pairs, there is a decomposition of the smash polyhedral product. Let

$$
J=\left\{j_{1}, j_{2}, \ldots, j_{k}\right\} \subset[m]
$$

and set $\widehat{B}^{J}=B_{j_{1}} \wedge B_{j_{2}} \wedge \cdots \wedge B_{j_{k}}$. Similarly, define $\widehat{C}^{J}$. 
Theorem $12.2([17])$. Let $(\underline{X}, \underline{A})=(\underline{B \vee C}, \underline{B \vee E})$ be a wedge decomposable pair. Then there is a homotopy equivalence

$$
\widehat{Z}(K ;(\underline{X}, \underline{A})) \longrightarrow \bigvee_{I \leq[m]} \widehat{Z}\left(K_{I} ;(\underline{C}, \underline{E})_{I}\right) \wedge \widehat{B}^{([m]-I)}
$$

which is natural with respect to maps of wedge decomposable pairs.

Since the inclusion $E_{i} \hookrightarrow C_{i}$ is null homotopic, the terms $\widehat{Z}\left(K_{I} ;(\underline{C}, \underline{E})_{I}\right)$ are completely determined by the the wedge lemma, [10], as follows.

Proposition 12.3. For pairs $(\underline{C}, \underline{E})$ as above there is a homotopy equivalence

$$
\widehat{Z}\left(K_{I} ;(\underline{C}, \underline{E})_{I}\right) \rightarrow \bigvee_{\sigma \in K_{I}}\left|1 \mathrm{k}_{\sigma}\left(K_{I}\right)\right| * \widehat{D}_{\underline{C}, \underline{E}}^{I}(\sigma)
$$

where $\left|\mathrm{lk}_{\sigma}\left(K_{I}\right)\right|$ is the realization of the link of $\sigma$ in the full subcomplex $K_{I}$ and

$$
\widehat{D}_{\underline{C}, \underline{E}}^{I}(\sigma)=\bigwedge_{j=1}^{|I|} W_{i_{j}}, \quad \text { with } \quad W_{i_{j}}= \begin{cases}C_{i_{j}} & \text { if } \quad i_{j} \in \sigma \\ E_{i_{j}} & \text { if } \quad i_{j} \in I-\sigma .\end{cases}
$$

Combined with the splitting Theorem 8.6, these results give a complete description of the topological spaces $Z(K ;(\underline{X}, \underline{A}))$ for wedge decomposable pairs $(\underline{X}, \underline{A})$.

The case $E_{i} \simeq *$ simplifies further by Theorem 8.11 to give the next corollary.

Corollary 12.4. For wedge decomposable pairs of the form $(\underline{B \vee C}, \underline{B})$, corresponding to $E_{i} \simeq *$ for all $i=1,2, \ldots, m$, there are homotopy equivalences

$$
\widehat{Z}\left(K_{I} ;(\underline{C}, \underline{E})_{I}\right) \simeq \widehat{Z}\left(K_{I} ;(\underline{C}, *)_{I}\right) \simeq \widehat{C}^{I},
$$

and so Theorem 12.2 gives $\widehat{Z}(K ;(\underline{B \vee C}, \underline{B})) \simeq \bigvee_{I \subseteq[m]}\left(\widehat{C}^{I} \wedge \widehat{B}^{([m]-I)}\right)$.

Notice that the Poincaré series for the space $\widehat{Z}(K ;(\underline{B \vee C}, \underline{B}))$ follows easily from Corollary 12.4 .

Remark. In comparing these observations with Theorem 11.3 , notice that the links appear in the terms $\widehat{Z}\left(K_{I} ;(\underline{C}, \underline{E})_{I}\right)$. Also, while Theorem 12.2 and Proposition 12.3 give a geometric underpinning for the cohomology calculation in Theorem 11.3 for wedge decomposable pairs only, the geometric splitting does not require that $E, B$ or $C$ have torsion-free cohomology

Theorem 12.2 applies particularly well in cases where spaces have unstable attaching maps.

Example 12.5. The homotopy equivalence $S^{1} \wedge Y \simeq \Sigma(Y)$ implies homotopy equivalences

$$
\Sigma^{m q}(\widehat{Z}(K ;(\underline{X}, \underline{A}))) \longrightarrow \widehat{Z}\left(K ;\left(\underline{\Sigma^{q}(X)}, \underline{\Sigma^{q}(A)}\right)\right)
$$


where as usual, $m$ is the number of vertices of $K$. Next, recalling that $S O(3) \cong \mathbb{R P}^{3}$, consider the pair

$$
(X, A)=\left(S O(3), \mathbb{R P}^{2}\right)
$$

for which there is a well known homotopy equivalence of pairs

$$
\left(\Sigma^{2}(S O(3)), \Sigma^{2}\left(\mathbb{R P}^{2}\right)\right) \longrightarrow\left(\Sigma^{2}\left(\mathbb{R P}^{2}\right) \vee \Sigma^{2}\left(\mathrm{~S}^{3}\right), \Sigma^{2}\left(\mathbb{R P}^{2}\right)\right)
$$

making the pair $\left(S O(3), \mathbb{R P}^{2}\right)$ stably wedge decomposable. Now, combining $(12.2)$ and (12.3), we get a homotopy equivalence

$$
\Sigma^{2 m}\left(\widehat{Z}\left(K ;\left(S O(3), \mathbb{R} \mathrm{P}^{2}\right)\right)\right) \longrightarrow \widehat{\mathrm{Z}}\left(\mathrm{K} ;\left(\Sigma^{2}\left(\mathbb{R} \mathrm{P}^{2}\right) \vee \Sigma^{2}\left(\mathrm{~S}^{3}\right), \Sigma^{2}\left(\mathbb{R} \mathrm{P}^{2}\right)\right) .\right.
$$

Finally, Theorem 12.2 allows us to conclude that $\left.\widehat{Z}\left(K ;\left(S O(3), \mathbb{R P}^{2}\right)\right)\right)$, and hence the polyhedral product $Z\left(K ;\left(S O(3), \mathbb{R P}^{2}\right)\right)$, is stably a wedge of smash products of $S^{3}$ and $\mathbb{R P}^{2}$. Similar splitting results follow for the polyhedral product whenever the spaces $X$ and $A$ split after finitely many suspensions. In particular, the fact that $\Omega^{2} S^{3}$ splits stably into a wedge of Brown-Gitler spectra implies that the polyhedral product $Z\left(K ;\left(\Omega^{2} S^{3}, *\right)\right)$ splits stably into a wedge of smash products of Brown-Gitler spectra.

The result of the previous section can be exploited to give information about the groups $H^{*}(\widehat{Z}(K ;(\underline{X}, \underline{A})))$ over a field $k$ for pointed, finite, path connected finite pairs of $\mathrm{CW}$ complexes $(\underline{X}, \underline{A})$, which are $\boldsymbol{n o t}$ wedge decomposable. In so doing, we explain further the remark in Example 11.6 .

Given $(\underline{X}, \underline{A})$, let $B_{i}, C_{i}$ and $E_{i}$ be the $k$-modules specified in items (1) and (2) of Definition 11.2. Now, wedges of spheres and Moore spaces $B_{i}^{\prime}, C_{i}^{\prime}$ and $E_{i}^{\prime}$ exist realizing the modules $B_{i}, C_{i}$ and $E_{i}$ so that

$$
\left(\underline{B^{\prime} \vee C^{\prime}}, \underline{B^{\prime} \vee E^{\prime}}\right)
$$

satisfies the criterion for a wedge decomposable pair as in Definition 12.1. Moreover, the diagram below commutes:

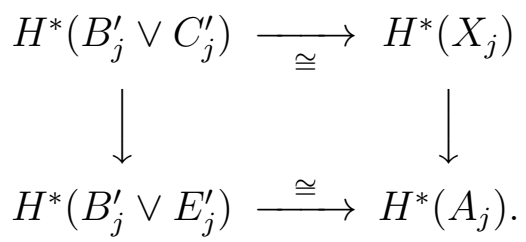

This leads to the following result.

Theorem 12.6 ([17]). Under the conditions stated above, the following isomorphism of groups holds for cohomology with coefficients in a field $k$

$$
H^{*}(\widehat{Z}(K ;(\underline{X}, \underline{A}))) \cong H^{*}\left(\widehat{Z}\left(K ;\left(\underline{B^{\prime} \vee C^{\prime}}, \underline{B^{\prime} \vee E^{\prime}}\right)\right)\right)
$$

where the right hand side is determined by Theorem 12.2 and Proposition 12.3. (This is not necessarily an isomorphism of modules over the Steenrod algebra.) 
As a consequence, the additive structure of $H^{*}(Z(K ;(X, A)) ; k)$ over a field $k$, is a functor which is determined by $H^{*}(X), H^{*}(A)$, and the ranks of the restriction maps appearing in the extension

$$
0 \rightarrow V_{i} \rightarrow H^{i}(X) \rightarrow H^{i}(A) \rightarrow W_{i} \rightarrow 0
$$

(cf. [134]). Information about the ring structure requires additional assumptions.

\section{Polyhedral products and the Golodness of monomial ideal Rings}

We begin with the definition of a Golod ring, [60, 89].

Definition 13.1. Let $S=k\left[x_{1}, x_{2}, \ldots, x_{m}\right]$ be a polynomial ring in $m$ variables over a field $k$, and let $I=\left(m_{1}, m_{2}, \ldots, m_{r}\right)$ be an ideal generated by monomials. The monomial ideal ring $R=S / I$ is called Golod if

$$
\sum_{j=0}^{\infty} \operatorname{dim} \operatorname{Tor}_{j}^{R}(k, k) t^{j}=\frac{(1+t)^{m}}{1-t\left(\sum_{j=0}^{\infty} \operatorname{dim}_{\operatorname{Tor}_{j}^{S}}(R, k) t^{j}-1\right)}
$$

Remark. According to [89] and [66], Serre had observed that the coefficients on the left hand side in (13.1) are always less than or equal to the corresponding coefficients on the right.

In [66], Golod showed that (13.1) holds if and only if all products and all higher Massey products vanish in $\operatorname{Tor}^{S}(R, K)$, (see also [89]). Higher Massey products in $H^{*}(X ; \mathbb{Q})$ obstruct the rational formality of $X$. As is noted in [96], this has a bearing on whether a complex manifold can admit a Kähler structure, an observation about the formality of Kähler manifolds which can be found in the paper by P. Deligne, Ph. A. Griffiths, J. W. Morgan, and D. Sullivan [54]. Golodness is relevant also in symplectic geometry and the theory of subspace arrangements; an overview of these connections can be found in [55] and [96].

The study of Golodness is particularly important in the case that $R$ is the StanleyReisner ring $k(K)$ of a simplicial complex $K$, (see (5.1)). The property has been much investigated by algebraic combinatorial theorists. The arrival of moment-angle complexes has invigorated this line of research. Moment-angle complexes become relevant via the split fibration arising from Theorem 10.1, namely

$$
\Omega Z\left(K ;\left(D^{2}, S^{1}\right)\right) \longrightarrow \Omega Z\left(K ;\left(\mathbb{C P}^{\infty}, *\right)\right) \longrightarrow T^{m}
$$

As noted in Section 5, there is an isomorphism of rings

$$
H^{*}\left(Z\left(K ;\left(D^{2}, S^{1}\right)\right)\right) \cong \operatorname{Tor}_{S}^{*}(k(K), k) .
$$


Using these ideas, and the isomorphism

$$
H^{*}\left(\Omega Z\left(K ;\left(\mathbb{C P}^{\infty}, *\right)\right) \cong \operatorname{Tor}_{k(K)}^{*}(k, k),\right.
$$

due to Buchstaber and Panov [35], in conjunction with an Eilenberg-Moore spectral sequence argument, J. Grbić and S. Theriault, [69, Theorem 11.1], were able to recapture for Stanley-Reisner rings the inequality involving the terms in (13.1), mentioned in the remark above and attributed to Serre in the general case. Moreover, extending Theorem 8.3, they showed that for a certain class $\mathcal{F}_{0}$, of simplicial complexes, obtained from shifted simplicial complexes by elementary topological operations, $Z\left(K ;\left(D^{2}, S^{1}\right)\right)$ has the homotopy type of a wedge of spheres, implying Golodness. G. Denham and A. Suciu also study obstructions to Golodness, and in particular, triple Massey products in lowest degree in [55].

Theorem 13.2. [69, Theorem 1.2]. If $K \in \mathcal{F}_{0}$, then $k(K)$ is a Golod ring over $k$.

K. Iriye and D. Kishimoto [82, begin with the stable decomposition of Theorem 8.12 applied to the case $(\underline{C X}, \underline{X})$ to get

$$
\Sigma Z(K ;(\underline{C X}, \underline{X})) \simeq \Sigma \bigvee_{\varnothing \neq I \subset[m]} \Sigma K_{I} \wedge \widehat{X}^{I} .
$$

They notice that if 13.4 ) desuspends for $X=S^{1}$, the space $Z\left(K ;\left(D^{2}, S^{1}\right)\right)$ remains a suspension and hence (13.3) implies that $k(K)$ is Golod over $k$, [118, Corollary 3.11].

They introduce a structure on $Z\left(K ;\left(D^{1}, S^{0}\right)\right)$ which they call the fat wedge filtration. This then allows them to give sufficient conditions on $K$ ensuring the desuspension of (13.4) for any $X$ and concluding that $k(K)$ is a Golod ring. Among these results is the next theorem.

Theorem 13.3. [82] If the Alexander dual of $K$ is shellable, then $Z\left(K ;\left(D^{n}, S^{n-1}\right)\right)$ has the homotopy type of a wedge of spheres, and hence $k(K)$ is Golod over $k$.

They are able also to recover a result of Herzog, Reiner and Welker.

Theorem 13.4. [77, 82] If the Alexander dual of the simplicial complex $K$ is sequentially Cohen-Macaulay over $k$, then $k(K)$ is Golod over $k$.

Fat wedge filtration techniques are also employed by K. Iriye and D. Kishimoto to prove that if $K$ is a triangulated surface orientable over $k$, then $k(K)$ is Golod over $k$ if and only if it is $2-n e i g h b o r l y$, that is, any two vertices are connected by an edge, [80, Theorem 1.3]. Using similar ideas, K. Iriye and D. Kishimoto [81] find a two-dimensional simplicial complex $K$ with $k(K)$ Golod over $\mathbb{Q}$ but not over $\mathbb{Z} / p$.

Remark. The fat wedge filtration methods of K. Iriye and D. Kishimoto [82] allow them to recover a variety of stable and unstable homotopy decompositions of moment-angle complexes and other polyhedral products. 
K. Iriye and T. Yano [84], also take the approach of desuspending (13.4). They use a simplicial complex constructed from a triangulated Hopf map $\eta: S^{3} \rightarrow S^{2}$, and Alexander duality to prove the existence of a simplicial complex $K$ for which $k(K)$ is a Golod ring but $Z\left(K ;\left(D^{2}, S^{1}\right)\right)$ is not a suspension.

J. Grbić, T. Panov, S. Theriault and J. Wu give an example, [72, Example 3.3], of a simplicial complex $K$, the standard 6-vertex triangulation of $\mathbb{R P}^{2}$, which is Golod over any field but $Z\left(K ;\left(D^{2}, S^{1}\right)\right)$ is not a wedge of spheres because it has torsion. I. Limonchenko gives a similar example arising from a 9-vertex triangulation of $\mathbb{C P}^{2}$, [95, Theorem 2.5]. He does this by explicitly computing the Betti numbers of $Z\left(K ;\left(D^{2}, S^{1}\right)\right)$ using (5.3), and then checking that the attaching maps in the stable splitting Corollary 8.13 are all stable maps. In [96], I. Limonchenko determines conditions on the bigraded Betti numbers (5.3) which imply the non-Golodness of $K$ over $k$. He uses the simplicial wedge construction (6.2), to find a family of generalized moment-angle complexes, such that for any $l, r \geq 2$, the family contains an $l$-connected manifold $M$ with a non-trivial $r$-fold Massey product in $H^{*}(M, \mathbb{Q})$.

Another approach to the Golodness problem for $K$ via the homotopy theory of the moment-angle complex $Z\left(K ;\left(D^{2}, S^{1}\right)\right)$, can be found in the work of P. Beben and J. Grbić, [25]. A. Berglund gives a combinatorial condition in [26] which ensures the Golodness of a monomial ideal ring.

\section{Higher WhiteheAd PRODUCTS AND LOOP SPACES}

Higher Whitehead products were introduced into the homotopy theory of momentangle complexes in the work of T. Panov and N. Ray [112]. They were concerned with the problem of realizing sphere wedge summands in $Z\left(K ;\left(D^{2}, S^{1}\right)\right)$ by higher Whitehead products. This work is summarized in [36, Sections 8.4 and 8.5]. Cases of this problem are discussed also in [71] and [83]. Recently, S. Abramyan [1] showed that not all sphere summands can be realized in this way using higher Whitehead products.

In the language of moment-angle complexes, the maps of 1.2 can be reformulated as follows. Let $K$ be the simplicial complex consisting of two discrete vertices, the boundary of a one-simplex $\partial \Delta^{1}$, and consider the corresponding fibration (10.1),

$$
Z\left(\partial \Delta^{1} ;\left(D^{2}, S^{1}\right)\right) \stackrel{\widetilde{\omega}}{\longrightarrow} Z\left(K ;\left(\mathbb{C P}^{\infty}, *\right)\right) \longrightarrow \mathbb{C P}^{\infty} \times \mathbb{C P}^{\infty} .
$$

We have

$$
\begin{aligned}
Z\left(\partial \Delta^{1} ;\left(D^{2}, S^{1}\right)\right) & =S^{1} \times D^{2} \cup_{S^{1} \times S^{1}} D^{2} \times S^{1} \simeq S^{3} \\
Z\left(\partial \Delta^{1} ;\left(S^{2}, *\right)\right) & =\left(S^{2} \times *\right) \cup_{* \times *}\left(* \times S^{2}\right)=S^{2} \vee S^{2} \\
Z\left(\partial \Delta^{1} ;\left(\mathbb{C P} P^{\infty}, *\right)\right) & =\mathbb{C P}^{\infty} \vee \mathbb{C P}^{\infty} \\
\left.Z\left(\Delta^{1} ;(\mathbb{C P})^{\infty}, *\right)\right) & =\mathbb{C P}^{\infty} \times \mathbb{C P}^{\infty}
\end{aligned}
$$


Then (14.1) factors as

$$
S^{3} \simeq Z\left(\partial \Delta^{1} ;\left(D^{2}, S^{1}\right)\right) \longrightarrow Z\left(\partial \Delta^{1} ;\left(S^{2}, *\right)\right) \longrightarrow Z\left(\partial \Delta^{1} ;\left(\mathbb{C P}^{\infty}, *\right)\right) \longrightarrow \mathbb{C P}^{\infty} \times \mathbb{C P}^{\infty} .
$$

Here, the first map is induced by the map of pairs $\left(D^{2}, S^{1}\right) \rightarrow\left(S^{2}, *\right)$, the second by the inclusion $\left.\left(S^{2}, *\right) \rightarrow(\mathbb{C P})^{\infty}, *\right)$, and the third by the inclusion of simplicial complexes $\partial \Delta^{1} \hookrightarrow \Delta^{1}$.

Now suppose that $\partial \Delta^{k-1}$ is a minimal missing face of a simplicial complex $K$, then we get the generalization

$$
\begin{aligned}
S^{2 k-1} \simeq Z\left(\partial \Delta^{k-1} ;\left(D^{2}, S^{1}\right)\right) \longrightarrow & Z\left(\partial \Delta^{k-1} ;\left(S^{2}, *\right)\right) \\
& \longrightarrow Z\left(\partial \Delta^{k-1} ;\left(\mathbb{C P}^{\infty}, *\right)\right) \longrightarrow Z\left(K ;\left(\mathbb{C P} P^{\infty}, *\right)\right) .
\end{aligned}
$$

Here, the last map is induced by the inclusion $\partial \Delta^{k-1} \hookrightarrow K$ and $Z\left(\partial \Delta^{k-1} ;\left(\mathbb{C P}^{\infty}, *\right)\right)$ retracts off $Z\left(K ;\left(\mathbb{C P}^{\infty}, *\right)\right)$ by [55, Proposition 3.3.1]. Following [36, page 339], the group $\pi_{2}\left(Z\left(K ;\left(\mathbb{C P}^{\infty}, *\right)\right)\right) \cong \mathbb{Z}^{m}$ has $m$ generators

$$
\widehat{\mu}_{i}: S^{2} \rightarrow \mathbb{C P}^{\infty} \stackrel{i}{\rightarrow} \mathbb{C P}^{\infty} \vee \mathbb{C P}^{\infty} \vee \cdots \vee \mathbb{C P}^{\infty} \rightarrow Z\left(K ;\left(\mathbb{C P}^{\infty}, *\right)\right),
$$

where the second map is the inclusion of the $i^{\text {th }}$ wedge summand and the last map is induced by the inclusion of the zero-skeleton into $K$. Next, labelling the vertices of the missing face $\partial \Delta^{k-1}$ by $\left\{i_{1}, i_{2}, \ldots, i_{k}\right\}$, we call the composite map (14.2) the $k$-fold higher Whitehead product and denote it by the symbol $\left[\widehat{\mu}_{i_{1}}, \widehat{\mu}_{i_{2}}, \ldots, \widehat{\mu}_{i_{k}}\right]_{w}$.

These maps, and associated Samelson products, play an important role in the study of the homotopy theory of $\Omega Z\left(K ;\left(D^{2}, S^{1}\right)\right)$ and $\Omega Z\left(K ;\left(\mathbb{C P}{ }^{\infty}, *\right)\right)$, particularly cases for which $Z\left(K ;\left(D^{2}, S^{1}\right)\right)$ is homotopy equivalent to a wedge of spheres, see [36, Section 8.4]. In the case of a flag complex $K, \mathrm{~T}$. Panov and N. Ray [112] compute the rational Pontrjagin ring of $\Omega Z\left(K ;\left(\mathbb{C P}^{\infty}, *\right)\right)$ by introducing various algebraic and geometric models. Motivated in part by this, and the work of S. Papadima and A. Suciu [116], N. Dobrinskaya [56] also addresses $\Omega Z(K ;(X, *))$ from a different point of view, and relates the computation to diagonal arrangements. (Recall from Section 8 that $Z\left(K ;\left(D^{2}, S^{1}\right)\right.$ ) is a deformation retract of $Z\left(K ;\left(\mathbb{C}, \mathbb{C}^{*}\right)\right)$, the complement of a complex coordinate arrangement determined by $K$.)

K. Iriye and D. Kishimoto, [83], call a simplicial complex $K$ totally fillable if each of its full subcomplexes has the property that it becomes contractible if some of its minimal missing faces are added. They employ their theory of fat wedge filtrations [82, to show that if $K$ is totally fillable, the moment-angle complex $Z\left(K ;\left(D^{2}, S^{1}\right)\right)$ decomposes as a wedge of spheres. For each such sphere $S^{\alpha}$, they recognize the map

$$
S^{\alpha} \hookrightarrow Z\left(K ;\left(D^{2}, S^{1}\right)\right) \stackrel{\widetilde{\omega}}{\longrightarrow} Z\left(K ;\left(\mathbb{C P}^{\infty}, *\right)\right)
$$

in terms of higher and iterated Whitehead product, [83, Theorem 1.3]. 
K. Iriye and D. Kishimoto then extend the discussion, as do J. Grbić and S. Theriault in [71], to the more general fibration

$$
Z(K ;(C(\Omega \underline{X})), \Omega \underline{X})) \stackrel{\widetilde{\omega}}{\longrightarrow} Z(K ;(\underline{X}, *)) \longrightarrow \prod_{i=1}^{m} X_{i},
$$

(which was studied also by G. Porter, [118]). In both papers, the map $\widetilde{\omega}$ is described fully in particular cases. More comprehensive information about Whitehead products in the context of 14.3 is to be found in the paper by S. Theriault [126]. The rational type of the fibre in 14.3) is studied also in the paper of Y. Félix and D. Tanré [59].

Acknowledgements. The authors would like to thank Haynes Miller, Peter Landweber, Taras Panov and Jelena Grbić for their careful reading of the manuscript and for their many valuable suggestions. Our thanks also to Santiago López de Medrano, Daisuke Kishimoto, Matthias Franz, Alex Suciu, Alvise Trevisan, Mentor Stafa, Stephen Theriault, Kouyemon Iriye and Graham Denham, for comments and recommendations which have led to a discernible improvement in the presentation.

\section{REFERENCES}

[1] S. Abramyan, Iterated higher Whitehead poducts in topology of moment-angle complexes. Online at: https://arxiv.org/pdf/1708.01694.pdf

[2] A. Al-Raisi, Branched covers, Strickland maps and cohomology related to the polyhedral product functor, Thesis, University of Rochester, (2014)

[3] A. Al-Raisi, F. R. Cohen and E. Vidaurre, Automorphisms of polyhedral products and their applications, in preparation.

[4] D. Allen and J. La Luz, Local face rings and diffeomorphisms of quasitoric manifolds, Homology, Homotopy and Applications, 21(1), 2019, 1-20.

[5] D. Anick, Connections between Yoneda and Pontrjagin algebras, Algebraic Topology, Aarhus 1982, Springer-Verlag Lecture Notes in Math., 1051 (1984), 331-350.

[6] L. Astey, A. Bahri, M. Bendersky, F. Cohen, D. Davis, S. Gitler, M. Mahowald, N. Ray and R. Wood, The $K O^{*}$-rings of $B T^{m}$, the Davis-Januszkiewicz spaces and certain toric manifolds. Journal of Pure and Applied Algebra, 218, (2014), 303-320.

[7] M. F. Atiyah, Convexity and commuting Hamiltonians, Bull. London Math. Soc. 14. (1982), 1-15, doi:10.1112/blms/14.1.1

[8] A. Ayzenberg, Substitutions of polytopes and of simplicial complexes, and multigraded Betti numbers, Trans. Moscow Math. Soc. (2013), 175-202 DOI: 10.1090/S0077-1554-2014-00224-7

[9] A. Bahri and M. Bendersky, The KO-theory of toric manifolds. Transactions of the American Mathematical Society, 352, (2000), 1191-1202.

[10] A. Bahri, M. Bendersky, F. R. Cohen, and S. Gitler, Decompositions of the polyhedral product functor with applications to moment-angle complexes and related spaces, PNAS, July, 2009, 106:12241-12244. 
[11] A. Bahri, M. Bendersky, F. Cohen and S. Gitler, The Polyhedral Product Functor: a method of computation for moment-angle complexes, arrangements and related spaces. Advances in Mathematics, 225 (2010), 1634-1668.

[12] A. Bahri, M. Bendersky, F. Cohen and S. Gitler, Cup products in generalized moment-angle complexes. Mathematical Proceedings of the Cambridge Philosophical Society, 153, (2012), 457-469.

[13] A. Bahri, M. Bendersky, F. Cohen and S. Gitler, The geometric realization of monomial ideal rings and a theorem of Trevisan, Homology, Homotopy and Applications, 14(1), (2012), 1-8.

[14] A. Bahri, M. Bendersky, F. Cohen and S. Gitler, A generalization of the Davis-Januszkiewicz construction and applications to toric manifolds and iterated polyhedral products, "Perspectives in Lie Theory", F. Callegaro, G. Carnovale, F. Caselli, C. De Concini, A. De Sole (Eds.), Springer INdAM series, (2017), 369-388.

[15] A. Bahri, M. Bendersky, F. Cohen and S. Gitler, Operations on polyhedral products and a new topological construction of infinite families of toric manifolds. Homology, Homotopy and Applications, 17, (2015), 137-160. Article available at http://dx.doi.org/10.4310/HHA.2015.v17.n2.a8

[16] A. Bahri, M. Bendersky, F. Cohen and S. Gitler, A spectral sequence for polyhedral products. Advances in Mathematics, 308, (2017), 767-814

[17] A. Bahri, M. Bendersky, F. Cohen and S. Gitler, A Cartan formula for polyhedral products, in preparation

[18] A. Bahri, S. Sarkar and J. Song, Infinite families of equivariantly formal toric orbifolds, to appear in Forum Mathematicum. Online at: https://arxiv.org/pdf/1801.04094.pdf

[19] A. Bahri, S. Sarkar and J. Song, On the integral cohomology ring of toric orbifolds and singular toric varieties, Algebraic and Geometric Topology, 17(6), 3779-3810

[20] A. Bahri, D. Notbohm, S. Sarkar and J. Song, On the integral cohomology of certain orbifolds, to appear in International Mathematics Research Notices. https://dx.doi.org/10.1093/imrn/rny283

[21] Y. Barreto, S. López de Medrano and A.Verjovsky, Some open book and contact structures on moment-angle manifolds,. Boletín de la Sociedad Matemática Mexicana 23, (2017), 423-437.

[22] V. Baskakov, Cohomology of $K$-powers of spaces and the combinatorics of simplicial divisions, Russian Math. Surveys, 57 (2002), no. 5, 989-990.

[23] I. Baskakov, V. Buchstaber and T. Panov, Cellular cochain complexes and torus actions, Uspeckhi. Mat. Nauk, 59, (2004), no. 3, 159-160 (russian); Russian Math. Surveys 89, (2004), no. 3, 562-563 (English translation).

[24] L. W. Beineke and F. Harary, The genus of the n-cube, Canadian Journal of Mathematics, 17, (1965), 494-496.

[25] P. Beben and J. Grbić, J. Configuration spaces and polyhedral products. Advances in Mathematics, (2017), 378-425. DOI: 10.1016/j.aim.2017.05.001

[26] A. Berglund, Poincaré series and homotopy Lie algebras of monomial rings, Research Report, no. 6, Stockholm University, (2005)

[27] E. Bifet, C. De Concini and C. Procesi, Cohomology of regular embeddings, Advances in Mathematics, 82, (1990), 1-34.

[28] A. Björner and M. L. Wachs, Shellable nonpure complexes and posets II, Trans. AMS 349, (1997), $3945-3975$. 
[29] A. Björner, M. L. Wachs, and V. Welker, On sequentially Cohen-Macaulay complexes and posets, Israel J. Math. 169, (2009), 295-316.

[30] F. Bosio and L. Meersseman, Real quadrics in $\mathbb{C}^{n}$, complex manifolds and convex polytopes, Acta Math., 197 (2006), 53-127, DOI: 10.1007/s11511-006-0008-2

[31] M. Brion, Piecewise polynomial functions, convex polytopes and enumerative geometry, P. Pragacz (ed.), Parameter Spaces. Banach Cent. Publ. 36 (Warszawa 1996), 25-44.

[32] K. Brown, Cohomology of Groups, GTM 87 Springer, (1982).

[33] W. Bruns and J. Gubeladze, Combinatorial invariance of Stanley-Reisner rings, Georgian Mathematical Journal, 3(4), (1996), 315-318.

[34] V. Buchstaber, and T. Panov, Actions of tori, combinatorial topology and homological algebra, Russian Math. Surveys, 55 (2000), no. 5, 825-921.

[35] V. Buchstaber and T. Panov, Torus actions and their applications in topology and combinatorics, AMS University Lecture Series, 24, (2002).

[36] V. Buchstaber and T. Panov, Toric Topology, AMS Mathematical Surveys and Monographs, 204 (2015).

[37] V. Buchstaber, T. Panov and N. Ray, Spaces of polytopes and cobordism of quasitoric manifolds, Moscow Math. Journal 7 (2007), no. 2, 219-242.

[38] V. Buchstaber and N. Ray, An invitation to toric topology: vertex four of a remarkable tetrahedron. In: Toric Topology (M. Harada et al., eds.). Contemp. Math., 460, Amer. Math. Soc., Providence, RI, (2008), 1-27.

[39] V. Buchstaber, N. Erokhovets, M. Masuda, T. Panov and S. Park, Cohomological rigidity of manifolds defined by right-angled 3-dimensional polytopes, Uspekhi Mat. Nauk 72(2), (2017), 3-66 (Russian); Russian Math. Surveys 72(2), (2017), 199-256 (English translation); DOI:10.1070/RM9759.

[40] L. Cai, On products in a real moment-angle manifold, J. Math. Soc. Japan, 69(2), (2017), 503-528.

[41] L. Cai and S. Choi, Integral cohomology groups of real toric manifolds and small covers. Online at: https://arxiv.org/pdf/1604.06988.pdf

[42] X. Cao and Z. Lü, Möbius transform, moment-angle complexes and Halperin-Carlsson conjecture, Journal of Algebraic Combinatorics, 35, (2012),121-140 DOI 10.1007/s10801-011-0296-2

[43] R. Charney and M. Davis, The K( $\pi, 1)$-problem for hyperplane complements associated to infinite reflection groups, Journal of the AMS 8, (1995), 597-627.

[44] S. Cho, S. Choi and S. Kaji, Geometric representations of finite groups on real toric spaces. Online at: https://arxiv.org/abs/1704.08591

[45] S. Choi and H. Park, Wedge operations and torus symmetries, Tohoku Math. J. (2), 68(1) (2016), 91-138.

[46] Y. Civan and N. Ray, Homotopy decompositions and real K-Theory of Bott towers, $K$-Theory 34, (2005), 1-33.

[47] F. R. Cohen and F. P. Peterson, Suspensions of Stiefel manifolds, Quart. J. Math. Oxford Ser. (2), 35(138), (1984), 115-119.

[48] A. Connes, Noncommutative differential geometry, Inst. Hautes Études Sci. Publ. Math., 62, (1985), 257-360. 
[49] H. S. M. Coxeter, Regular skew polyhedra in three and four dimension, and their topological analogues, Proc. London Math. Soc. (1938) s2-43 (1), 33-62.

[50] S. Das, Genus of the hypercube graph and real moment-angle complex. Online at: https://arxiv.org/abs/1806.10220

[51] M. W. Davis, Groups generated by reflections and aspherical manifolds not covered by Euclidean space, Ann. of Math. 117, (1983), no. 2, 293-324.

[52] M. Davis and T. Januszkiewicz, Convex polytopes, Coxeter orbifolds and torus actions, Duke Math. J., 62, (1991), no. 2, 417-451.

[53] M. Davis and B. Okun, Cohomology computations for Artin groups, Bestvina-Brady groups, and graph products, Groups Geom. Dyn. 6, (2012), 485-531 Bull. Soc. Math. France 116, (1988), no. 3, 315-339.

[54] P. Deligne, Ph. A. Griffiths, J. W. Morgan, and D. Sullivan, Real homotopy theory of Kähler manifolds, Invent. Math. 29, (1975), 245-274.

[55] G. Denham and A. Suciu, Moment-angle complexes, monomial ideals and Massey products, Pure Appl. Math. Q., 3 (2007), no. 1, 25-60.

[56] N. Dobrinskaya, Loops on polyhedral products and diagonal arrangements. Online at: https://arxiv.org/abs/0901.2871

[57] S. Eilenberg and J. A. Zilber, Semi-simplicial complexes and singular homology, Annals of Mathematics, Second Series, 51 (1950), 499-513.

[58] N. Erokhovets, Buchstaber invariant of simple polytopes, Uspekhi Mat. Nauk 63(5), (2008), 187-188 (Russian); Russian Math. Surveys 63(5), (2008), 962-964, (English translation).

[59] Y. Félix and D. Tanré, Rational homotopy of the polyhedral product functor, Proceedings of the American Mathematical Society,. 137, (2009), 891-898.

[60] R. Frankhuizen, $A^{\infty}$-resolutions and the Golod property for monomial rings. Online at: https://arxiv.org/abs/1612.02737

[61] M. Franz, On the integral cohomology of smooth toric varieties. Online at: https://arxiv.org/format/math/0308253

[62] M. Franz, The integral cohomology of toric manifolds, Proc. Steklov Inst. Math., 252 (2006), 53-62, [Proceedings of the Keldysh Conference, Moscow 2004].

[63] X. Fu and J. Grbić, Simplicial G-complexes and representational stability of polyhedral products. Online at: https://arxiv.org/abs/1803.11047

[64] T. Ganea, A generalization of the homology and homotopy suspension, Comment. Math. Helv., 39 (1965), 295-322.

[65] S. Gitler and S. López de Medrano, Intersections of quadrics, moment-angle manifolds and connected sums, Geometry \& Topology 17 (3), 1497-1534.

[66] E. S. Golod, On the cohomology of some local rings, (Russian); Soviet Math. Dokl. 3, (1962), 745749 .

[67] M. Goresky and R. MacPherson, Stratified Morse Theory, Ergebnisse der Mathematik und ihrer Grenzgebiete 3rd series, 14, Springer-Verlag, Berlin, 1988.

[68] J. Grbić and S. Theriault, Homotopy type of the complement of a coordinate subspace arrangement of codimension two, Russian Math. Surveys 59 (2004), no. 3, 1207-1209. 
[69] J. Grbić and S. Theriault, The homotopy type of the complement of a coordinate subspace arrangement, Topology 46, (2007), 357-396.

[70] J. Grbic and S. Theriault, The homotopy type of the polyhedral product for shifted complexes, Advances in Mathematics, 245, (2013) 690-715. DOI: 10.1016/j.aim.2013.05.002

[71] J. Grbić and S. Theriault, Homotopy theory in toric topology, Russian Math. Surveys 71, (2016), $185-251$.

[72] J. Grbić, T. Panov, S. Theriault and J. Wu, (2016). The homotopy types of moment-angle complexes for flag manifolds, Transactions of the American Mathematical Society, 368(9), 6663-6682. DOI: $10.1090 / \operatorname{tran} / 6578$

[73] V. Grujić and V. Welker, Moment-angle complexes of pairs $\left(D^{n}, S^{n-1}\right)$ and simplicial complexes with vertex-decomposable duals, Monatsh. Math. 176(2), (2015), 255-273.

[74] A. Hatcher, Algebraic Topology, Cambridge Univ. Press, Cambridge, 2001.

[75] A. Hattori and M. Masuda, Theory of multi-fans, Osaka J. Math. 40, (2003) 1-68.

[76] G. C. Haynes, F. R. Cohen, and D. Koditschek, Gait Transitions for Quasi-Static Hexapedal Locomotion on Level Ground, 14-th International Symposium on Robotics Research (ISRR 2009).

[77] J. Herzog, V. Reiner, V. Welker, Componentwise linear ideals and Golod rings, Michigan Math. J. 46, (1999), 211-223.

[78] M. Hochster, Cohen-Macaulay rings, combinatorics, and simplicial complexes, In: Ring Theory II (Proc. Second Oklahoma Conference), B. R. McDonald and R. Morris, eds. Dekker, New York, (1977), 171-223.

[79] K. Iriye and D. Kishimoto, Decompositions of polyhedral products for shifted complexes, Advances in Mathematics, 245 (2013), 716-736.

[80] K. Iriye and D. Kishimoto, Golodness and polyhedral products for two dimensional simplicial complexes. Forum Math. 30(2), (2018), 527-532.

[81] K. Iriye and D. Kishimoto, Golodness and polyhedral products of simplicial complexes with minimal Taylor resolutions, Homology Homotopy Appl. 20(1), (2018), 69-78.

[82] K. Iriye and D. Kishimoto, Fat wedge filtrations and decomposition of polyhedral products, To appear in Kyoto J. Math. (DOI:10.1215/21562261-2017-0038)

[83] K. Iriye and D. Kishimoto, Whitehead products in moment-angle complexes. Online at: https://arxiv.org/pdf/1807.00087.pdf

[84] K. Iriye and T. Yano, A Golod complex with non-suspension moment-angle complex, Topology Appl. 225, (2017), 145-163.

[85] I. M. James, Reduced product spaces, Annals of Mathematics, 62, No. 1 (1955), 170-197.

[86] I. M. James, The topology of Stiefel manifolds, Cambridge University Press, (1977).

[87] J. Jurkiewicz, On the complex projective torus embedding, the associated variety with corners and Morse functions, Bull. Acad. Pol. Sci., Sr. Sci. Math. 29, (1981), 21-27.

[88] Y. Kamiyama and S. Tsukuda, The configuration space of the $n$-arms machine in the Euclidean space, Topology and its Applications, 154 (2007), 1447-1464.

[89] L. Katthän, A non-Golod ring with a trivial product on its Koszul homology, J. Algebra, 479 (2017), 244-262 
[90] K. H. Kim and F. W. Roush, Homology of certain algebras defined by graphs, J. Pure Appl. Algebra 17, (1980), 179-186.

[91] D. Kishimoto, Right-angled Coxeter quandles and polyhedral products. Online at: arXiv:1706.06209v2

[92] A. Kurosh, Lectures on general algebra, published in Russian in 1960 and an English translation published in 1963, the Chelsea Publishing Company, New York.

[93] H. Kuwata, M. Masuda, H. Zeng, Torsion in the cohomology of torus orbifolds, Chinese Annals of Mathematics, 38(6), (2016), 1247-1268.

[94] J. La Luz and D. Allen, Certain generalized higher derived functors associated to quasitoric manifolds, Journal of Homotopy and Related Structures, DOI 10.1007/s40062-017-0186-y

[95] I. Limonchenko, Families of minimally non-Golod complexes and their polyhedral products, Far Eastern Mathematical Journal, 15:2, (2015), 222-237.

[96] I. Limonchenko, On higher Massey products and rational formality for moment-angle manifolds over multiwedges, to appear in Proc. Steklov Inst. Math., (2019). Online at: arXiv:1711.00461v2.

[97] I. Limonchenko, Bigraded Betti numbers of certain simple polytopes, Mathematical Notes 3(3), (2013) DOI: $10.1134 / \mathrm{S} 000143461309006 \mathrm{X}$

[98] J. L. Loday, Cyclic Homology, Springer, (1998).

[99] J. L. Loday and D. Quillen, Cyclic homology and the Lie algebra homology of matrices, Commentarii Mathematici Helvetici, 59, (1984), 565-591.

[100] S. López de Medrano, Topology of the intersection of quadrics in $\mathbb{R}^{n}$, Algebraic Topology, Arcata California, (1986), Lecture Notes in Mathematics, 1370 Springer-Verlag, (1989), 280-292.

[101] S. López de Medrano and A. Verjovsky, A new family of complex, compact, non-symplectic manifolds Bol. Soc. Bras. Mat., 28(2), (1997), 253-269

[102] Z. Lü and T. Panov, Moment-angle complexes from simplicial posets Central European Journal of Mathematics, 9(4), (2011), 715-730, DOI: 10.2478/s11533-011-0041-z

[103] L. Meersseman, A new geometric construction of compact complex manifolds in any dimension, Math. Ann., 317, (2000), 79-115.

[104] L. Meersseman and A. Verjovsky, Holomorphic principal bundles over projective topic varieties, J. Reine Angew. Math. 572, (2004), 57-96.

[105] H. Miller, Stable splittings of Stiefel manifolds, Topology 24(4), (1985), 411-419.

[106] J. Milnor, On the construction F[K], A Student's Guide to Algebraic Topology, J. F. Adams, editor, Lecture Notes of the London Mathematical Society, 4(1972), 119-136. Also in: Collected Papers of John Milnor IV, Homotopy, Homology and Manifolds, John McCleary editor, AMS 2009, 45-53.

[107] C. Moreau, Sur les permutations circulaires distinctes, Nouvelles Annales de Mathématiques, journal des candidats aux écoles polytechnique et normale, Sér. 2, 11, (1872) 309-331.

[108] D. Notbohm and N. Ray, On Davis-Januszkiewicz homotopy types I; formality and rationalisation, Algebr. Geom. Topol. 5 (2005) 31-51. DOI:10.2140/agt.2005.5.31

[109] T. Panov, Cohomology of face rings, and torus actions, London Math. Soc. Lecture Notes, volume 347, Surveys in Contemporary Mathematics, Cambridge, 2008, 165-201.

[110] T. Panov, Geometric structures on moment-angle manifolds, Uspekhi Mat. Nauk 68(3), (2013), 111-186 (Russian); Russian Math. Surveys 68(3), (2013), 503-568 (English translation) 
[111] T. Panov, N. Ray, and R. Vogt Colimits, Stanley-Reisner algebras, and loop spaces, in: Categorical decomposition techniques in algebraic topology, Progress in Mathematics, 215 (2004), 261-291, Birkhäuser, Basel.

[112] T. Panov and N. Ray, Categorical aspects of toric topology, Toric Topology, Contemp. Math. 460, Amer. Math. Soc. (2008), 293-322.

[113] T. Panov and S. Theriault, The homotopy theory of polyhedral products associated with flag complexes. Accepted for publication in Compositio Mathematica. Online at: https://arxiv.org/abs/1709.00388

[114] T. Panov and Y. Veryovkin, Polyhedral products and commutator subgroups of right-angled Artin and Coxeter groups, Mat. Sbornik, 207(11), (2016), 105-126 (Russian); Sbornik Math. 207(11), (2016), 1582-1600.

[115] T. Panov and Y. Ustinovsky. Complex-analytic structures on moment-angle manifolds. Moscow Math. J. 12 (2012), no. 1, 149-172.

[116] S. Papadima and A. Suciu, Algebraic invariants for right-angled Artin groups, Math. Annalen, 334(3), (2006), 533-555.

[117] G. Porter, The homotopy groups of wedges of suspensions, Amer. J. Math., 88 (1966), 655-663.

[118] G. Porter, Higher products, Transactions of the Amer. Math. Soc., 148, (1970), 315-345.

[119] J. S. Provan and L. J. Billera, Decompositions of simplicial complexes related to diameters of convex polyhedra, Mathematics of Operations Research, volume 5, (1980), 576-594.

[120] G. Ringel, Über drei kombinatorische Probleme am n-dimensionalen Würfel und Würfelgitter, Abhandlungen aus dem Mathematischen Seminar der Universität Hamburg, 20(1), (1955), 10-19.

[121] M. Stafa, Polyhedral products, flag complexes and monodromy representations, Topology Appl. 244, (2018), 12-30.

[122] M. Stafa, On the fundamental group of certain polyhedral products, J. Pure Appl. Algebra 219, (2015), no. 6, 2279-2299.

[123] N. Strickland, Notes on toric spaces, preprint 1999, (unpublished)

[124] A. Suciu, The rational homology of real toric manifolds, Oberwolfach Reports 9(4) (2012). 29722976.

[125] S. Theriault, Moment-angle manifolds and Panov's problem, International Mathematics Research Notices, Volume 2015, Issue 20, 10154-10175, https://doi.org/10.1093/imrn/rnu281

[126] S. Theriault, The dual polyhedral product, cocategory and nilpotence. Accepted for publication in Advances in Mathematics. Online at: https://arxiv.org/pdf/1506.05998.pdf

[127] A. Trevisan, Generalized Davis-Januszkiewicz spaces and their applications to algebra and topology, Thesis, Vrije Universiteit Amsterdam, (2012)

[128] Y. Ustinovsky, Doubling operation for polytopes and torus actions. Uspekhi Mat. Nauk, 64(5), (2009), 181-182 (Russian); Russian Math. Surveys 64(5), (2009), 952-954 (English).

[129] Y. Ustinovsky, Toral rank conjecture for moment-angle complexes, Mat. Zametki 90(2), (2011), 300-305 (Russian); Math. Notes 90(1-2), (2011), 279-283 (English). 5 (1971), 1083-1119.

[130] C. T. C. Wall, Stability, pencils and polytopes, Bull. London Math. Soc., 12, (1980), 401-421.

[131] V. Welker, G. Ziegler and R. Živaljević, Homotopy colimits-comparison lemmas for combinatorial applications, J. Reine Angew. Math., 509 (1999), 117-149. 
[132] E. Witt, Treue Darstellungen Lieschen Ringe, J. Reine Angew. Math,, 177, (1937), 152-160.

[133] L. Yu, On lower bounds of the sum of multigraded Betti numbers of simplicial complexes. Online at: https://arxiv.org/abs/1811.04398

[134] Q. Zheng, The homology coalgebra and cohomology algebra of generalized moment-angle complexes, Journal of Pure and Applied Algebra, (2012).

[135] G. Ziegler and R. Živaljević, Homotopy types of sub-space arrangements via diagrams of spaces, Math. Ann. 295 (1993), 527-548.

Department of Mathematics, Rider University, Lawrenceville, NJ 08648, U.S.A.

E-mail address: bahri@rider.edu

Department of Mathematics CUny, East 695 Park Avenue New York, NY 10065, U.S.A.

E-mail address: mbenders@hunter.cuny.edu

Department of Mathematics, University of Rochester, Rochester, NY 14625, U.S.A.

E-mail address: cohf@math.rochester.edu 